Supporting Information for

\title{
Acceptorless Dehydrogenative Coupling of o-Aminobenzamides with the Activation of Methanol as a C1 Source for the Construction of \\ Quinazolinones
}

\author{
Feng Li, ${ }^{*},{ }^{\dagger}$ Lei Lu, ${ }^{\dagger}$ and Pengcheng Liu $^{\dagger}$ \\ *E-mail: fengli@njust.edu.cn
}
${ }^{\dagger}$ School of Chemical Engineering, Nanjing University of Science \& Technology, Nanjing 210094, P. R. China
${ }^{\ddagger}$ State Key Laboratory of Fine Chemicals, Dalian University of Technology, Dalian 116024, P. R. China

Table of Contents:

$\begin{array}{ll}\text { General Experimental Details } & \text { S2 }\end{array}$

Experimental procedure and analytical data of products $\quad$ S2-S11

Copies of ${ }^{1} \mathrm{H}$ NMR and ${ }^{13} \mathrm{C}$ NMR spectra of products $\quad$ S12-S53 
Experimental Details. Proton nuclear magnetic resonance $\left({ }^{1} \mathrm{H}\right.$ NMR) spectra were recorded at $500 \mathrm{MHz}$. Chemical shifts are reported in delta $(\delta)$ units, parts per million (ppm) downfield from tetramethylsilane or ppm relative to the center of the singlet at $2.5 \mathrm{ppm}$ for DMSO- $\mathrm{d}_{6}$. Coupling constants $J$ values are reported in Hertz (Hz), and the splitting patterns were designated as follows: $\mathrm{s}$, singlet; $\mathrm{d}$, doublet; $\mathrm{t}$, triplet; $\mathrm{m}$, multiplet; $\mathrm{b}$, broad. Carbon-13 nuclear magnetic resonance $\left({ }^{13} \mathrm{C}\right.$ NMR) spectra were recorded at $125 \mathrm{MHz}$. Chemical shifts are reported in delta ( $\delta$ ) units, ppm relative to the center of the triplet at $39.5 \mathrm{ppm}$ for DMSO- $\mathrm{d}_{6} \cdot{ }^{13} \mathrm{C}$ NMR spectra were routinely run with broadband decoupling.

$\left[\mathrm{Cp}^{*} \mathrm{IrCl}_{2}\right]_{2} \quad\left(\right.$ cat. $\quad$ 1) ${ }^{1} \quad[\operatorname{Ir}(\operatorname{cod}) \mathrm{Cl}]_{2} \quad\left(\right.$ cat. $\quad$ 2) ${ }^{2} \quad\left[\mathrm{Cp}^{2} \operatorname{Ir}\left(\mathrm{NH}_{3}\right)_{3}\right][\mathrm{Cl}]_{2} \quad$ (cat. 3), ${ }^{3}$ $[\mathrm{Cp} * \operatorname{Ir}(2-$ phenylpyridine- $k \mathrm{C}, \mathrm{N})] \mathrm{Cl} \quad($ cat. $\left.\quad 4),{ }^{4} \quad[\mathrm{Cp} * \operatorname{Ir}(\mathrm{bpy}) \mathrm{Cl})\right][\mathrm{Cl}] \quad$ (cat. 5) ${ }^{5}$ $\left[\mathrm{Cp} * \operatorname{Ir}(\right.$ bpy $\left.)\left(\mathrm{H}_{2} \mathrm{O}\right)\right][\mathrm{OTf}]_{2}$ (cat. 6), ${ }^{6}[\mathrm{Cp} *(2-(\mathrm{OH}) \mathrm{py})] \mathrm{Cl}_{2}$ (cat. 7$){ }^{7}\left[\mathrm{Cp}^{*}\left(6,6{ }^{\prime}-(\mathrm{OH})_{2}\right.\right.$ bpy) $][\mathrm{OTf}]_{2}$ (cat. 8) ${ }^{8}$ and $\left[\mathrm{Cp} * \operatorname{Ir}\left(2,2^{\prime}\right.\right.$-bpyO $\left.)\left(\mathrm{H}_{2} \mathrm{O}\right)\right](\text { cat. 9 })^{9}$ were synthesized according to previous reports.

General procedure for acceptorless dehydrogenative coupling of o-aminobenzamides with methanol for the construction of quinazolinones catalyzed by $\left[\mathrm{Cp}^{*} \operatorname{Ir}\left(2, \mathbf{2}^{\prime}-\mathrm{bpyO}\right)\left(\mathrm{H}_{2} \mathrm{O}\right)\right]$ : To an oven-dried, $5 \mathrm{~mL}$ microwave vial containing a stirrer bar were added $o$-aminobenzamides $\mathbf{1}$ (0.5 mmol), methanol 2 (0.5 mL), cat. 9 (2.7 mg, $0.005 \mathrm{mmol}, 1 \mathrm{~mol} \%)$ and $\mathrm{Cs}_{2} \mathrm{CO}_{3}(49 \mathrm{mg}, 0.15$ mmol, 0.3 equiv). The vial was placed in a focused, single-mode microwave synthesizer (Discover CEM, USA $)$ at $130{ }^{\circ} \mathrm{C}$ for $2 \mathrm{~h}(300 \mathrm{~W}$, sealed reaction vessel $)$. Then, the mixture was allowed to cool to ambient temperature, concentrated in vacuo and purified by flash column chromatography with hexanes/ethyl acetate to afford the corresponding products. All experiments outlined in the paper are safe under current reaction conditions.

quinazolin-4(3H)-one (3a) ${ }^{10}$<smiles>O=c1[nH]cnc2ccccc12</smiles>

White solid; 88\% yield (64 mg); mp 215-217 ${ }^{\circ} \mathrm{C} ;{ }^{1} \mathrm{H}$ NMR (500 MHz, DMSO-d $\left.\mathrm{d}_{6}\right) \delta 12.26$ (br s, 1H, NH), 8.12 (d, 1H, J=7.9 Hz, ArH), 8.09 (s, 1H, ArH), 7.81 (t, 1H, J=7.1 Hz, ArH), 7.67 (t, $1 \mathrm{H}, J=8.1 \mathrm{~Hz}, \mathrm{ArH}), 7.52(\mathrm{t}, 1 \mathrm{H}, J=7.7 \mathrm{~Hz}, \operatorname{ArH}) ;{ }^{13} \mathrm{C}\left\{{ }^{1} \mathrm{H}\right\} \mathrm{NMR}\left(125 \mathrm{MHz}, \mathrm{DMSO}-\mathrm{d}_{6}\right) \delta$ $160.7,148.7,145.3,134.2,127.2,126.7,125.8,122.6$.

7-methylquinazolin-4(3H)-one (3b) ${ }^{11}$ 
<smiles>Cc1ccc2c(=O)[nH]cnc2c1</smiles>

White solid, 87\% yield (70 mg); mp 237-238 ${ }^{\circ} \mathrm{C} ;{ }^{1} \mathrm{H}$ NMR (500 MHz, DMSO-d 6 ) $\delta 12.13$ (br s, 1H, NH), 8.05 (s, 1H, ArH), 8.00 (d, 1H, $J=7.8 \mathrm{~Hz}, \operatorname{ArH}), 7.46$ (s, 1H, ArH), 7.33 (d, 1H, $J=7.5$ $\mathrm{Hz}, \mathrm{ArH}), 2.45$ (s, $\left.3 \mathrm{H}, \mathrm{CH}_{3}\right) ;{ }^{13} \mathrm{C}\left\{{ }^{1} \mathrm{H}\right\} \mathrm{NMR}\left(125 \mathrm{MHz}, \mathrm{DMSO}-\mathrm{d}_{6}\right) \delta 160.8,148.9,145.7,144.6$, $128.0,126.7,125.6,120.2,21.3$.

6-methylquinazolin-4(3H)-one (3c) ${ }^{12}$<smiles>Cc1ccc2nc[nH]c(=O)c2c1</smiles>

Light yellow solid; 90\% yield (72 mg); mp 249-251 ${ }^{\circ} \mathrm{C} ;{ }^{1} \mathrm{H}$ NMR (500 MHz, DMSO-d 6 ) $\delta 12.13$ (br s, 1H, NH), 8.02 (s, 1H, ArH), $7.91(\mathrm{~s}, 1 \mathrm{H}, \mathrm{ArH}), 7.63(\mathrm{dd}, 1 \mathrm{H}, J=8.3 \mathrm{~Hz}$ and $1.9 \mathrm{~Hz}, \mathrm{ArH}$ ), $7.56(\mathrm{~d}, 1 \mathrm{H}, J=8.3 \mathrm{~Hz}, \mathrm{ArH}), 2.44\left(\mathrm{~s}, 3 \mathrm{H}, \mathrm{CH}_{3}\right) ;{ }^{13} \mathrm{C}\left\{{ }^{1} \mathrm{H}\right\} \mathrm{NMR}\left(125 \mathrm{MHz}, \mathrm{DMSO}-\mathrm{d}_{6}\right) \delta 160.9$, $146.8,144.8,136.4,135.6,127.1,125.2,122.4,20.8$.

6-methoxyquinazolin-4(3H)-one (3d) ${ }^{13}$<smiles>COc1ccc2nc[nH]c(=O)c2c1</smiles>

White solid; 91\% yield (80 mg); mp 243-244 ${ }^{\circ} \mathrm{C} ;{ }^{1} \mathrm{H}$ NMR (500 MHz, DMSO-d 6 ) $\delta 8.01$ (s, $1 \mathrm{H}$, $\operatorname{ArH}), 7.57$ (d, 1H, $J=8.9 \mathrm{~Hz}, \operatorname{ArH}), 7.49$ (d, 1H, $J=2.3 \mathrm{~Hz}, \mathrm{ArH}), 7.36(\mathrm{dd}, 1 \mathrm{H}, J=8.9 \mathrm{~Hz}$ and $3.3 \mathrm{~Hz}, \mathrm{ArH}), 3.85$ (s, $\left.3 \mathrm{H}, \mathrm{CH}_{3}\right) ;{ }^{13} \mathrm{C}\left\{{ }^{1} \mathrm{H}\right\} \mathrm{NMR}\left(125 \mathrm{MHz}, \mathrm{DMSO}-\mathrm{d}_{6}\right): \delta 162.0,157.4,144.9$, 143.6, 128.6, 123.5, 123.3, 105.7, 55.5.

\section{6,7-dimethoxyquinazolin-4(3H)-one (3e $)^{14}$}<smiles>COc1cc2nc[nH]c(=O)c2cc1OC</smiles>

Gray solid, 86\% yield (89 mg); mp 296-299 ${ }^{\circ} \mathrm{C} ;{ }^{1} \mathrm{H}$ NMR (500 MHz, DMSO-d 6 ) $\delta 12.07$ (br s, $1 \mathrm{H}$, NH), $7.98(\mathrm{~s}, 1 \mathrm{H}, \mathrm{ArH}), 7.44(\mathrm{~s}, 1 \mathrm{H}, \mathrm{ArH}), 7.12(\mathrm{~s}, 1 \mathrm{H}, \mathrm{ArH}), 3.90\left(\mathrm{~s}, 3 \mathrm{H}, \mathrm{OCH}_{3}\right), 3.86(\mathrm{~s}, 3 \mathrm{H}$, $\left.\mathrm{OCH}_{3}\right) ;{ }^{13} \mathrm{C}\left\{{ }^{1} \mathrm{H}\right\}$ NMR $\left(125 \mathrm{MHz}, \mathrm{DMSO}-\mathrm{d}_{6}\right) \delta 160.1,154.4,148.5,144.8,143.8,115.6,108.0$, 104.9, 55.9, 55.7. 
<smiles>O=c1[nH]cnc2c(F)cccc12</smiles>

Gray solid, 72\% yield (59 mg); mp 270-272 ${ }^{\circ} \mathrm{C} ;{ }^{1} \mathrm{H}$ NMR (500 MHz, DMSO-d 6 ) $\delta 12.47$ (br s, $1 \mathrm{H}$, NH), 8.14 (s, 1H, ArH), 7.93 (d, 1H, $J=8.0 \mathrm{~Hz}, \mathrm{ArH}), 7.68$ (t, $1 \mathrm{H}, J=9.3 \mathrm{~Hz}, \mathrm{ArH}), 7.52-7.48$ (m, $1 \mathrm{H}, \mathrm{ArH}) ;{ }^{13} \mathrm{C}\left\{{ }^{1} \mathrm{H}\right\} \mathrm{NMR}\left(125 \mathrm{MHz}, \mathrm{DMSO}-\mathrm{d}_{6}\right) \delta 162.2,156.4\left(\mathrm{~d}, J_{\mathrm{C}-\mathrm{F}}=251.5 \mathrm{~Hz}\right), 148.7,138.7$ $\left(\mathrm{d}, J_{\mathrm{C}-\mathrm{F}}=10.4 \mathrm{~Hz}\right), 125.8,124.8,121.6,118.7\left(\mathrm{~d}, J_{\mathrm{C}-\mathrm{F}}=18.3 \mathrm{~Hz}\right)$.

6-fluoroquinazolin-4(3H)-one (3g)<smiles>O=c1[nH]cnc2ccc(F)cc12</smiles>

White solid, 77\% yield (63 mg); mp 252-255 ${ }^{\circ} \mathrm{C} ;{ }^{1} \mathrm{H}$ NMR (500 MHz, DMSO-d 6 ) $\delta 8.09$ (s, $1 \mathrm{H}$, ArH), $7.77(\mathrm{dd}, 1 \mathrm{H}, J=8.7 \mathrm{~Hz}$ and $2.9 \mathrm{~Hz}, \mathrm{ArH}), 7.73(\mathrm{dd}, 1 \mathrm{H}, J=8.9 \mathrm{~Hz}$ and $5.2 \mathrm{~Hz}, \mathrm{ArH}), 7.67$ $(\mathrm{td}, 1 \mathrm{H}, J=8.6 \mathrm{~Hz}$ and $3.0 \mathrm{~Hz}, \mathrm{ArH}) ;{ }^{13} \mathrm{C}\left\{{ }^{1} \mathrm{H}\right\} \mathrm{NMR}\left(125 \mathrm{MHz}, \mathrm{DMSO}-\mathrm{d}_{6}\right) \delta 160.6,159.9(\mathrm{~d}$, $\left.J_{\mathrm{C}-\mathrm{F}}=243.8 \mathrm{~Hz}\right), 145.7,145.4,129.9\left(\mathrm{~d}, J_{\mathrm{C}-\mathrm{F}}=7.5 \mathrm{~Hz}\right), 123.9\left(\mathrm{~d}, J_{\mathrm{C}-\mathrm{F}}=8.8 \mathrm{~Hz}\right), 122.6\left(\mathrm{~d}, J_{\mathrm{C}-\mathrm{F}}=\right.$ $23.8 \mathrm{~Hz}), 110.4\left(\mathrm{~d}, J_{\mathrm{C}-\mathrm{F}}=22.5 \mathrm{~Hz}\right)$.

\section{8-chloroquinazolin-4(3H)-one (3h) $)^{12}$}<smiles>O=c1[nH]cnc2c(Cl)cccc12</smiles>

Light yellow solid, 68\% yield (61mg); mp 296-298 ${ }^{\circ} \mathrm{C} ;{ }^{1} \mathrm{H}$ NMR (500 MHz, DMSO-d $\left.{ }_{6}\right) \delta 12.39$ (br s, 1H, NH), 8.21 (s, 1H, ArH), 8.08 (s, 1H, ArH), 7.97 (s, 1H, ArH), 7.49 (s, 1H, ArH) ${ }^{13} \mathrm{C}\left\{{ }^{1} \mathrm{H}\right\}$ NMR (125 MHz, DMSO-d $\left.\mathrm{d}_{6}\right) \delta 160.3,146.5,145.2,134.4,130.7,127.0,125.0,124.3$.

7-chloroquinazolin-4(3H)-one (3i) ${ }^{16}$<smiles>O=c1[nH]cnc2cc(Cl)ccc12</smiles>

White solid, 70\% yield (63 mg); mp 252-254 ${ }^{\circ} \mathrm{C} ;{ }^{1} \mathrm{H}$ NMR (500 MHz, DMSO-d 6 ) $\delta 12.44$ (br s, 
1H, NH), 8.13 (s, 2H, ArH), 7.72 (s, 1H, ArH), 7.55 (s, 1H, ArH); ${ }^{13} \mathrm{C}\left\{{ }^{1} \mathrm{H}\right\}$ NMR (125 MHz, DMSO-d $\left._{6}\right) \delta 160.4,149.9,147.2,138.8,127.9,126.9,126.3,121.4$.

6-chloroquinazolin-4(3H)-one (3j) $)^{15}$<smiles>O=c1[nH]cnc2ccc(Cl)cc12</smiles>

White solid, 77\% yield (70 mg); mp 263-265 ${ }^{\circ} \mathrm{C} ;{ }^{1} \mathrm{H}$ NMR (500 MHz, DMSO-d 6 ) $\delta 8.13$ (s, $1 \mathrm{H}$, ArH), $8.05(\mathrm{~d}, 1 \mathrm{H}, J=2.4 \mathrm{~Hz}, \mathrm{ArH}), 7.83(\mathrm{dd}, 1 \mathrm{H}, J=8.7 \mathrm{~Hz}$ and $2.4 \mathrm{~Hz}, \mathrm{ArH}), 7.69$ (d, 1H, $J=$ $8.7 \mathrm{~Hz}, \mathrm{ArH}) ;{ }^{13} \mathrm{C}\left\{{ }^{1} \mathrm{H}\right\}$ NMR $\left(125 \mathrm{MHz}, \mathrm{DMSO}-\mathrm{d}_{6}\right) \delta 160.0,147.5,146.2,134.3,130.9,129.4$, $124.8,123.9$.

\section{7-bromoquinazolin-4(3H)-one (3k) ${ }^{17}$}<smiles>O=c1[nH]cnc2cc(Br)ccc12</smiles>

Gray solid; 80\% yield (90 mg); mp 227-228 ${ }^{\circ} \mathrm{C} ;{ }^{1} \mathrm{H}$ NMR (500 MHz, DMSO-d 6 ) $\delta 8.16(\mathrm{~d}, 1 \mathrm{H}, J=$ $1.6 \mathrm{~Hz}, \mathrm{ArH}), 8.14$ (s, 1H, ArH), 7.87 (dd, 1H, $J=8.6 \mathrm{~Hz}$ and $1.6 \mathrm{~Hz}, \mathrm{ArH}), 7.56$ (d, 1H, $J=8.6$ $\mathrm{Hz}, \mathrm{ArH}) ;{ }^{13} \mathrm{C}\left\{{ }^{1} \mathrm{H}\right\}$ NMR $\left(125 \mathrm{MHz}, \mathrm{DMSO}-\mathrm{d}_{6}\right) \delta 161.7,148.6,148.2,136.2,129.2,127.9,124.3$, 118.1 .

6-bromoquinazolin-4(3H)-one (3I) ${ }^{18}$<smiles>O=c1[nH]cnc2ccc(Br)cc12</smiles>

White solid; 82\% yield (92 mg); mp 272-275 ${ }^{\circ} \mathrm{C} ;{ }^{1} \mathrm{H}$ NMR (500 MHz, DMSO-d 6 ) $\delta 12.39$ (br s, 1H, NH), 8.19 (s, 1H, ArH), 8.14 (s, 1H, ArH), 7.95 (d, 1H, $J=7.4 \mathrm{~Hz}, \mathrm{ArH}), 7.62$ (d, 1H, $J=8.6$ $\mathrm{Hz}, \mathrm{ArH}) ;{ }^{13} \mathrm{C}\left\{{ }^{1} \mathrm{H}\right\}$ NMR $\left(125 \mathrm{MHz}, \mathrm{DMSO}-\mathrm{d}_{6}\right) \delta 159.7,147.7,146.1,137.0,129.6,127.9,124.2$, 119.1 .

7-(trifluoromethyl)quinazolin-4(3H)-one (3m) $)^{11}$<smiles>O=c1[nH]cnc2cc(C(F)(F)F)ccc12</smiles> 
White solid, 78\% yield (84 mg); mp 226-228 ${ }^{\circ} \mathrm{C} ;{ }^{1} \mathrm{H}$ NMR (500 MHz, DMSO-d 6 ) $\delta 12.55$ (br s, $1 \mathrm{H}, \mathrm{NH}), 8.31$ (d, 1H, $J=8.5 \mathrm{~Hz}, \operatorname{ArH}), 8.23$ (s, 1H, ArH), 7.98 (s, 1H, ArH), 7.82 (d, 1H, $J=7.7$ $\mathrm{Hz}, \mathrm{ArH}) ;{ }^{13} \mathrm{C}\left\{{ }^{1} \mathrm{H}\right\} \mathrm{NMR}\left(125 \mathrm{MHz}, \mathrm{DMSO}-\mathrm{d}_{6}\right) \delta 160.1,148.8,147.2,133.9$ (q, $J_{\mathrm{C}-\mathrm{F}}=32.2 \mathrm{~Hz}$ ), $127.6,125.5,124.3,123.5\left(\mathrm{q}, J_{\mathrm{C}-\mathrm{F}}=269.7 \mathrm{~Hz}\right), 122.4$.

\section{3-butylquinazolin-4(3H)-one (3n) ${ }^{19}$}<smiles>CCCCn1cnc2ccccc2c1=O</smiles>

White solid; 66\% yield (67 mg); mp 70-72 ${ }^{\circ} \mathrm{C} ;{ }^{1} \mathrm{H}$ NMR (500 MHz, DMSO-d 6 ) $\delta 8.32$ (d, 1H, $J=$ $7.5 \mathrm{~Hz}, \mathrm{ArH}), 8.03$ (s, 1H, ArH), 7.76 (t, 1H, $J=7.3 \mathrm{~Hz}, \mathrm{ArH}), 7.71$ (d, 1H, $J=8.0 \mathrm{~Hz}, \mathrm{ArH}), 7.51$ (t, $1 \mathrm{H}, J=7.3 \mathrm{~Hz}, \mathrm{ArH}$ ), 4.01 (t, $2 \mathrm{H}, J=7.3 \mathrm{~Hz}, \mathrm{CH}_{2}$ ), 1.79 (quint, $2 \mathrm{H}, J=7.5 \mathrm{~Hz}, \mathrm{CH}_{2}$ ), 1.42 (sext, $\left.2 \mathrm{H}, J=7.4 \mathrm{~Hz}, \mathrm{CH}_{2}\right), 0.98\left(\mathrm{t}, 3 \mathrm{H}, J=7.3 \mathrm{~Hz}, \mathrm{CH}_{3}\right) ;{ }^{13} \mathrm{C}\left\{{ }^{1} \mathrm{H}\right\}$ NMR $(125 \mathrm{MHz}$, DMSO-d 6 ) $\delta 161.1,148.1,146.6,134.1,127.4,127.2,126.7,122.2,46.8,31.4,19.9,13.6$.

\section{$2 H$-benzo[e][1,2,4] thiadiazine 1,1-dioxide $(3 p)^{20}$}<smiles>O=S1(=O)NC=Nc2ccccc21</smiles>

White solid; 76\% yield (69 mg); mp 225-226 ${ }^{\circ} \mathrm{C} ;{ }^{1} \mathrm{H}$ NMR (500 MHz, DMSO-d 6 ) $\delta 12.15$ (br s, 1H, NH), 7.97 (s, 1H, ArH), 7.81 (d, 1H, J=7.9 Hz, ArH), 7.67 (t, 1H, J=7.7 Hz, ArH), 7.46 (t, $1 \mathrm{H}, J=7.6 \mathrm{~Hz}, \mathrm{ArH}), 7.32(\mathrm{~d}, 1 \mathrm{H}, J=8.3 \mathrm{~Hz}, \mathrm{ArH}) ;{ }^{13} \mathrm{C}\left\{{ }^{1} \mathrm{H}\right\} \mathrm{NMR}\left(125 \mathrm{MHz}, \mathrm{DMSO}^{-} \mathrm{d}_{6}\right) \delta$ $147.7,134.8,133.0,126.6,123.6,122.5,117.5$.

Procedure for the synthesis of 6,7-bis(2-methoxyethoxy)quinazolin-4(3H)-one (5). ${ }^{21}$ To an oven-dried, $5 \mathrm{~mL}$ microwave vial containing a stirrer bar were added 4 (142 mg, $0.5 \mathrm{mmol}$ ), methanol 2 (0.5 mL), cat. 9 (2.7 mg, $0.005 \mathrm{mmol}, 1 \mathrm{~mol} \%$ ) and $\mathrm{Cs}_{2} \mathrm{CO}_{3}(49 \mathrm{mg}, 0.15 \mathrm{mmol}, 0.3$ equiv). The vial was placed in a focused, single-mode microwave synthesizer (Discover CEM, USA) at $130{ }^{\circ} \mathrm{C}$ for $2 \mathrm{~h}(300 \mathrm{~W}$, sealed reaction vessel). Then, the mixture was allowed to cool to ambient temperature, concentrated in vacuo and purified by flash column chromatography with hexanes/ethyl acetate to afford the corresponding product $\mathbf{5}$.<smiles>COCCOc1cc2nc[nH]c(=O)c2cc1OCCOC</smiles> 
Light yellow solid; $85 \%$ yield (125 mg); mp 180-182 ${ }^{\circ} \mathrm{C} ;{ }^{1} \mathrm{H}$ NMR $(500 \mathrm{MHz}$, DMSO-d 6 ) $\delta 12.05$ (br s, 1H, NH), 7.97 (s, 1H, ArH), 7.46 (s, 1H, ArH), 7.15 (s, 1H, ArH), 4.25 (t, 2H, J=4.3 Hz, $\left.\mathrm{CH}_{2}\right), 4.19$ (t, $\left.2 \mathrm{H}, J=4.4 \mathrm{~Hz}, \mathrm{CH}_{2}\right), 3.71\left(\mathrm{~m}, 4 \mathrm{H}, 2 \mathrm{xCH}_{2}\right), 3.33\left(\mathrm{~s}, 6 \mathrm{H}, 2 \mathrm{xCH}_{3}\right) ;{ }^{13} \mathrm{C}\left\{{ }^{1} \mathrm{H}\right\} \mathrm{NMR}$ $\left(125 \mathrm{MHz}\right.$, DMSO-d $\left.\mathrm{d}_{6}\right) \delta 160.1,153.8,147.8,144.8,143.9,115.7,109.0,106.5,70.2,70.1,68.1$, $68.1,58.3$.

Procedure for the synthesis of 4-chloro-6,7-bis(2-methoxyethoxy)quinazoline (6). ${ }^{21} \mathbf{5}$ (328 mg, $1.1 \mathrm{mmol})$ was slowly added to a solution of phosphoryl chloride $(1 \mathrm{~mL})$ and $N, N$-diethylaniline (164 mg, $1.1 \mathrm{mmol}$ ). This mixture was heated at $90{ }^{\circ} \mathrm{C}$ for $2 \mathrm{~h}$ and was then cooled to room temperature. Most of the excess of phosphoryl chloride was removed under reduced pressure and the dark oil was purified by flash column chromatography with hexanes/ethyl acetate to afford the corresponding product.<smiles>COCCOc1cc2ncnc(Cl)c2cc1OCCOC</smiles>

White Solid, 83\% yield (286 mg), mp 106-107 ${ }^{\circ} \mathrm{C}:{ }^{1} \mathrm{H}$ NMR (500 MHz, DMSO-d 6 ) $\delta 8.86(\mathrm{~s}, 1 \mathrm{H}$, ArH), 7.44 (s, 1H, ArH), 7.33 (s, 1H, ArH), 4.35-4.32 (m, 4H, 2xCH $), 3.90-3.88$ (m, 4H, 2xCH $\mathrm{xCH}_{2}$, 3.51 (s, $\left.3 \mathrm{H}, \mathrm{CH}_{3}\right), 3.49$ (s, $\left.3 \mathrm{H}, \mathrm{CH}_{3}\right) ;{ }^{13} \mathrm{C}\left\{{ }^{1} \mathrm{H}\right\} \mathrm{NMR}\left(125 \mathrm{MHz}, \mathrm{DMSO}-\mathrm{d}_{6}\right) \delta 158.9,156.2,152.4$, $150.8,148.9,119.4,107.6,104.0,70.4,70.2,68.8,68.7,59.3\left(2 \mathrm{xOCH}_{3}\right)$.

Procedure for the synthesis of $N$-(3-ethynylphenyl)-6,7-Bis-(2-methoxyethoxy)-quinazolin-4amine (7, Erlotinib). ${ }^{21}$ A solution of $6(286 \mathrm{mg}, 0.91 \mathrm{mmol})$ in $i$-PrOH ( $\left.5 \mathrm{~mL}\right)$ was added drop wise to a solution of pyridine $(79 \mathrm{mg}, 1.0 \mathrm{mmol})$ and 3-ethynylphenylamine $(117 \mathrm{mg}, 1.0 \mathrm{mmol})$ in $i$-PrOH $(5 \mathrm{~mL})$. This mixture was heated at reflux for $4 \mathrm{~h}$ under nitrogen and was then cooled to room temperature. The precipitate was filtered and crystallized from methanol to afford a $7 \cdot \mathrm{HCl}$ product. $7 \cdot \mathrm{HCl}$ product was dissolved in water, basified using conc. aq. ammonia, and extracted with ethyl acetate and concentrated in vacuo to give free 7.<smiles>C#Cc1cccc(Nc2ncnc3cc(OCCOC)c(OCCOC)cc23)c1</smiles>

Yellowish Solid, 90\% yield (323 mg); mp 156-157 ${ }^{\circ} \mathrm{C} ;{ }^{1} \mathrm{H}$ NMR (500 MHz, DMSO-d $\left.\mathrm{d}_{6}\right) \delta 8.61$ (s, 1H, ArH), 7.99 (s, 1H, ArH), 7.85 (s, 1H, ArH), 7.74 (dd, 1H, $J=8.1 \mathrm{~Hz}$ and $1.0 \mathrm{~Hz}, \mathrm{ArH}), 7.30$ (t, 
$1 \mathrm{H}, J=7.9 \mathrm{~Hz}, \mathrm{ArH}), 7.23(\mathrm{~d}, 1 \mathrm{H}, J=7.7 \mathrm{~Hz}, \mathrm{ArH}), 4.18-4.14\left(\mathrm{~m}, 4 \mathrm{H}, 2 \mathrm{xCH}_{2}\right), 3.77-3.73(\mathrm{~m}, 4 \mathrm{H}$, $\left.2 \mathrm{xCH}_{2}\right), 3.40\left(\mathrm{~s}, 3 \mathrm{H}, \mathrm{CH}_{3}\right), 3.39$ (s, $\left.3 \mathrm{H}, \mathrm{CH}_{3}\right), 3.08(\mathrm{~s}, 1 \mathrm{H}, \mathrm{CH}) ;{ }^{13} \mathrm{C}\left\{{ }^{1} \mathrm{H}\right\}$ NMR $(125 \mathrm{MHz}$, DMSO-d $\left.\mathrm{d}_{6}\right) \delta 156.4,154.2,153.5,148.5,147.3,138.9,128.9,127.6,125.2,122.6,122.4,109.2$, $108.4,102.5,83.4,77.4,70.8,70.3,68.8,68.1,59.1,59.1$.

Procedure for acceptorless dehydrogenative coupling of o-aminobenzamides with other alcohols catalyzed by metal-organic bifunctional iridium complex: To an oven-dried, $5 \mathrm{~mL}$ microwave vial containing a stirrer bar were added $o$-aminobenzamide $1 \mathrm{a}(68 \mathrm{mg}, 0.5 \mathrm{mmol})$, alcohols 8-10 (0.5 mL), cat. 9 (2.7 mg, $0.005 \mathrm{mmol}, 1 \mathrm{~mol} \%)$ and $\mathrm{Cs}_{2} \mathrm{CO}_{3}(49 \mathrm{mg}, 0.15 \mathrm{mmol}, 0.3$ equiv). The vial was placed in a focused, single-mode microwave synthesizer (Discover CEM, USA) at $130{ }^{\circ} \mathrm{C}$ for $2 \mathrm{~h}(300 \mathrm{~W}$, sealed reaction vessel). Then, the mixture was allowed to cool to ambient temperature, concentrated in vacuo and purified by flash column chromatography with hexanes/ethyl acetate to afford the corresponding products.

2-methylquinazolin-4(3H)-one (11)<smiles>Cc1nc2ccccc2c(=O)[nH]1</smiles>

White solid; 90\% yield (72 mg); mp 242-243 ${ }^{\circ} \mathrm{C} ;{ }^{1} \mathrm{H}$ NMR (500 MHz, $\mathrm{CDCl}_{3}$ ) $\delta 11.85$ (br s, $1 \mathrm{H}$, $\mathrm{NH}), 8.28$ (d, 1H, $J=7.5 \mathrm{~Hz}, \operatorname{ArH}), 7.78$ (t, 1H, $J=7.1 \mathrm{~Hz}, \operatorname{ArH}), 7.68(\mathrm{~d}, 1 \mathrm{H}, J=8.0 \mathrm{~Hz}, \operatorname{ArH})$, $7.48(\mathrm{t}, 1 \mathrm{H}, J=7.5 \mathrm{~Hz}, \mathrm{ArH}), 2.60\left(\mathrm{~s}, 3 \mathrm{H}, \mathrm{CH}_{3}\right) ;{ }^{13} \mathrm{C}\left\{{ }^{1} \mathrm{H}\right\} \mathrm{NMR}\left(125 \mathrm{MHz}, \mathrm{CDCl}_{3}\right) \delta 164.5,153.4$, $149.5,134.8,127.0,126.3,126.2,120.2,22.0$.

\section{2-ethylquinazolin-4(3H)-one $(12)^{23}$}<smiles>CCc1nc2ccccc2c(=O)[nH]1</smiles>

White solid; $86 \%$ yield $\left(75 \mathrm{mg}\right.$ ); $\mathrm{mp} 232-233{ }^{\circ} \mathrm{C} ;{ }^{1} \mathrm{H}$ NMR $\left(500 \mathrm{MHz}, \mathrm{CDCl}_{3}\right) \delta 11.67$ (br s, $1 \mathrm{H}$, $\mathrm{NH}), 8.29(\mathrm{~d}, 1 \mathrm{H}, J=7.1 \mathrm{~Hz}, \mathrm{ArH}), 7.77$ (t, 1H, $J=8.3 \mathrm{~Hz}, \mathrm{ArH}), 7.72(\mathrm{~d}, 1 \mathrm{H}, J=8.0 \mathrm{~Hz}, \operatorname{ArH})$, $7.47(\mathrm{t}, 1 \mathrm{H}, J=7.8 \mathrm{~Hz}, \mathrm{ArH}), 2.85\left(\mathrm{q}, 2 \mathrm{H}, J=7.6 \mathrm{~Hz}, \mathrm{CH}_{2}\right), 1.46\left(\mathrm{t}, 3 \mathrm{H}, J=7.6 \mathrm{~Hz}, \mathrm{CH}_{3}\right) ;{ }^{13} \mathrm{C}$ $\left\{{ }^{1} \mathrm{H}\right\}$ NMR $\left(125 \mathrm{MHz}, \mathrm{CDCl}_{3}\right) \delta 164.4,157.8,149.4,134.7,127.2,126.3,126.2,120.4,29.0$, 11.5 .

2-phenyl-4(3H)-quinazolinone (13) $)^{24}$ 
<smiles>O=c1[nH]c(-c2ccccc2)nc2ccccc12</smiles>

White solid; 91\% yield (111 mg); mp 220-221 ${ }^{\circ} \mathrm{C} ;{ }^{1} \mathrm{H}$ NMR (500 MHz, $\left.\mathrm{CDCl}_{3}\right) \delta 10.27$ (br s, $1 \mathrm{H}$, NH), 8.33 (d, 1H, $J=8.1 \mathrm{~Hz}, \operatorname{ArH}), 8.14-8.12(\mathrm{~m}, 2 \mathrm{H}, \operatorname{ArH}), 7.85-7.79(\mathrm{~m}, 2 \mathrm{H}, \operatorname{ArH}), 7.59(\mathrm{~d}, 3 \mathrm{H}$, $J=7.0 \mathrm{~Hz}, \mathrm{ArH}), 7.52(\mathrm{t}, 1 \mathrm{H}, J=7.9 \mathrm{~Hz}, \mathrm{ArH}) ;{ }^{13} \mathrm{C}\left\{{ }^{1} \mathrm{H}\right\} \mathrm{NMR}\left(125 \mathrm{MHz}, \mathrm{CDCl}_{3}\right) \delta 164.0,151.8$, $149.5,134.8,132.8,131.6,129.0,128.0,127.5,126.7,126.3,120.8$.

Procedure for the hydrogen evolution experiment. ${ }^{25} \mathbf{1 a}(68 \mathrm{mg}, 0.5 \mathrm{mmol}), 2(0.5 \mathrm{~mL})$, cat. 9 (2.7 mg, $1 \mathrm{~mol} \%$ ) and $\mathrm{Cs}_{2} \mathrm{CO}_{3}$ (49 $\mathrm{mg}, 0.15 \mathrm{mmol}, 0.3$ equiv) were added into a $5 \mathrm{~mL}$ thick walled glass vessel fitted with a side arm and a rubber septum through a valve. The vessel was previously degassed three times and placed under a $\mathrm{N}_{2}$ atmosphere. The vessel was connected to the gas collection apparatus (standard water displacement apparatus, using a graduated cylinder to determine volume) through a valve and the entire system was flushed with $\mathrm{N}_{2}$ for 5 min and allowed to equilibrate for $5 \mathrm{~min}$. The valve was closed and the reaction mixture was stirred vigorously at $150{ }^{\circ} \mathrm{C}$ for $12 \mathrm{~h}$ under sealed conditions. When reaction mixture was cooled to room temperature and the valve was turned on and the gas was collected by water displacement until gas evolution ceased.

The GC analysis was performed on a gas chromatograph with TCD detector. Injector temprature $=150{ }^{\circ} \mathrm{C}$, column temprature $=80{ }^{\circ} \mathrm{C}$, detector temperature $(\mathrm{TCD})=80{ }^{\circ} \mathrm{C}$, carrier gas $=\mathrm{N}_{2}$, column flow $=20 \mathrm{~mL} / \mathrm{min}, t=0.525 \mathrm{~min}$.

The volume of $1 \mathrm{~mol}$ of $\mathrm{H}_{2}$ at $283.15 \mathrm{~K}, 101810 \mathrm{~Pa}$ was calculated according to the van der Waals equation as shown below

$$
\left(p+\frac{n^{2} a}{V^{2}}\right)(V-n b)=n R T
$$

where $R=8.3145 \mathrm{~m}^{3} \mathrm{~Pa} \cdot \mathrm{mol}^{-1} \cdot \mathrm{K}^{-1} ; T=283.15 \mathrm{~K} ; p=102600 \mathrm{~Pa} ; a=0.02476 \mathrm{~m}^{6} \cdot \mathrm{Pa} \cdot \mathrm{mol}^{-2} ; b=$ $0.02661 \times 10^{-3} \mathrm{~m}^{3} \cdot \mathrm{mol}^{-1}$; thus, $V\left(\mathrm{H}_{2}, 283.15 \mathrm{~K}, 102600 \mathrm{~Pa}\right)=23 \mathrm{~L} \cdot \mathrm{mol}^{-1}$.

The collected volume of gas in this experiment above was $14 \mathrm{~mL}$, which corresponds to 0.61 mmol of $\mathrm{H}_{2}$. 
Procedure for acceptorless dehydrogenative coupling of o-aminobenzamides with paraformaldehyde catalyzed by $\left[\mathbf{C p *} \operatorname{Ir}\left(2,2^{\prime}-\mathbf{b p y O}\right)\left(\mathbf{H}_{2} \mathrm{O}\right)\right]$ : To an oven-dried, $5 \mathrm{~mL}$ microwave vial containing a stirrer bar were added $o$-aminobenzamide $1 \mathrm{a}(0.5 \mathrm{mmol}, 68 \mathrm{mg})$, paraformaldehyde $(45 \mathrm{mg})$, toluene $(1 \mathrm{~mL})$, cat. 9 (2.7 mg, $0.005 \mathrm{mmol}, 1 \mathrm{~mol} \%)$ and $\mathrm{Cs}_{2} \mathrm{CO}_{3}(49$ $\mathrm{mg}, 0.15 \mathrm{mmol}, 0.3$ equiv). The vial was placed in a focused, single-mode microwave synthesizer (Discover CEM, USA) at $130{ }^{\circ} \mathrm{C}$ for $2 \mathrm{~h}(300 \mathrm{~W}$, sealed reaction vessel). Then, the mixture was allowed to cool to ambient temperature, concentrated in vacuo and purified by flash column chromatography with hexanes/ethyl acetate to afford the corresponding product $\mathbf{3 a}$ (60 $\mathrm{mg}, 82 \%$ yield).

\section{References}

(1) Ball, R. G.; Graham, W. A. G.; Heinekey, D. M.; Hoyano, J. K.; McMaster, A. D.; Mattson, B. M.; Michel, S. T. Inorg. Chem. 1990, 29, 2023-2025.

(2) Choudhury, J.; Podder, S.; Roy, S. J. Am. Chem. Soc. 2005, 127, 6162-6163.

(3) Kawahara, R.; Fujita, K.; Yamaguchia, R. Adv. Synth. Catal. 2011, 353, 1161-1168.

(4) Scheeren, C.; Maasarani, F.; Hijazi, A.; Djukic, J. P.; Pfeffer, M.; Zaric, S. D.; Goff, X. F. L.; Ricard, L. Organometallics 2007, 26, 3336-3345.

(5) Ogo, S.; Makihara, N.; Kaneko, Y.; Watanabe, Y. Organometallics 2001, 20, 4903-4910.

(6) Ziessel, R. J. Chem. Soc., Chem. Commun. 1988, 16-17.

(7) Fujita, K.; Tanino, N.; Yamaguchi, R. Org. Lett. 2007, 9, 109-111.

(8) Kawahara, R.; Fujita, K.; Yamaguchi, R. J. Am. Chem. Soc. 2012, 134, 3643-3646.

(9) Kawahara, R.; Fujita, K.; Yamaguchi, R. Angew. Chem. Int. Ed. 2012, 51, 12790-12794.

(10) Yang, X.; Cheng, G.; Shen, J.; Kuai, C.; Cui, X. Org. Chem. Front. 2015, 2, 366-368.

(11) Kulkarni, S. S.; Singh, S.; Shah, J. R.; Low, W.; Talele, T. T. Eur. J. Med. Chem. 2012, 50, 264-273.

(12) Nara, H.; Sato, K.; Naito, T.; Mototani, H.; Oki, H.; Yamamoto, Y.; Kuno, H.; Santou, T.; Kanzaki, N.; Terauchi, J.; Uchikawa, O.; Kori, M. Bioorg. Med. Chem. 2014, 22, 5487-5505. 
(13) LeMahieu, R. A.; Carson, M.; Nason, W. C.; Parrish, D. R.; Welton, A. F.; Baruth, H. W.; Yaremko, B. J. Med. Chem. 1983, 26, 420-425.

(14) VanBrocklin, H. F.; Lim, J. K.; Coffing, S. L.; Hom, D. L.; Negash, K.; Ono, M. Y.; Gilmore, J. L.; Bryant, I.; Riese, D. J. J. Med. Chem. 2005, 48, 7445-7456.

(15) Pedgaonkar, G. S.; Sridevi, J. P.; Jeankumar, V. U.; Saxena, S.; Devi, P. B.; Renuka, J.; Yogeeswari, P.; Sriram, D. Eur. J. Med. Chem. 2014, 86, 613-627.

(16) Luo, H.; Yang, S.; Cai, Y.; Peng, Z.; Liu, T. Eur. J. Med. Chem. 2014, 84, 746-752.

(17) Hedou, D.; Guillon, R.; Lecointe, C.; Logec, C.; Chosson, E.; Besson, T. Tetrahedron 2013, $69,3182-3191$.

(18) Gao, L.; Kovackova, S.; Sala, M.; Ramadori, A. T.; Jonghe, S. D.; Herdewijn, P. J. Med. Chem. 2014, 57, 7624-7643.

(19) Bao, Y.; Yan, Y.; Xu, K.; Su, J.; Zha, Z.; Wang, Z. J. Org. Chem. 2015, 80, 4736-4742.

(20) Chen, X.; Zhu, C.; Guo, F.; Qiu, X.; Yang, Y.; Zhang, S.; He, M.; Parveen, S.; Jing, C.; Li, Y.; Ma, B. J. Med. Chem. 2010, 53, 8330-8344.

(21) Knesl, P.; Röseling, D.; Jordis, U. Molecules 2006, 11, 286-297.

(22) Ding, P.; Gao, M.; Mao, B.; Cao, S.; Liu, C.; Yang, C.; Li, Z.; Liao, J.; Zhao, H.; Li, Z.; Li, J.; Wang, H.; Xu, X. Eur. J. Med. Chem. 2016, 108, 364-373.

(23) Xu, W.; Fu, H. J. Org. Chem. 2011, 76, 3846-3852.

(24) Zhao, W.; Liu, P.; Li, F. ChemCatChem 2016, 8, 1523-1530.

(25) Wu, J.; Talwar, D.; Johnston, S.; Yan, M.; Xiao, J. Angew. Chem. Int. Ed. 2013, 52, 6983-6987. 


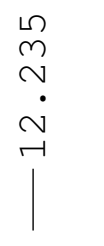

걱유

m न

o o o

$\longrightarrow$<smiles>O=c1[nH]cnc2ccccc12</smiles>

$3 a$
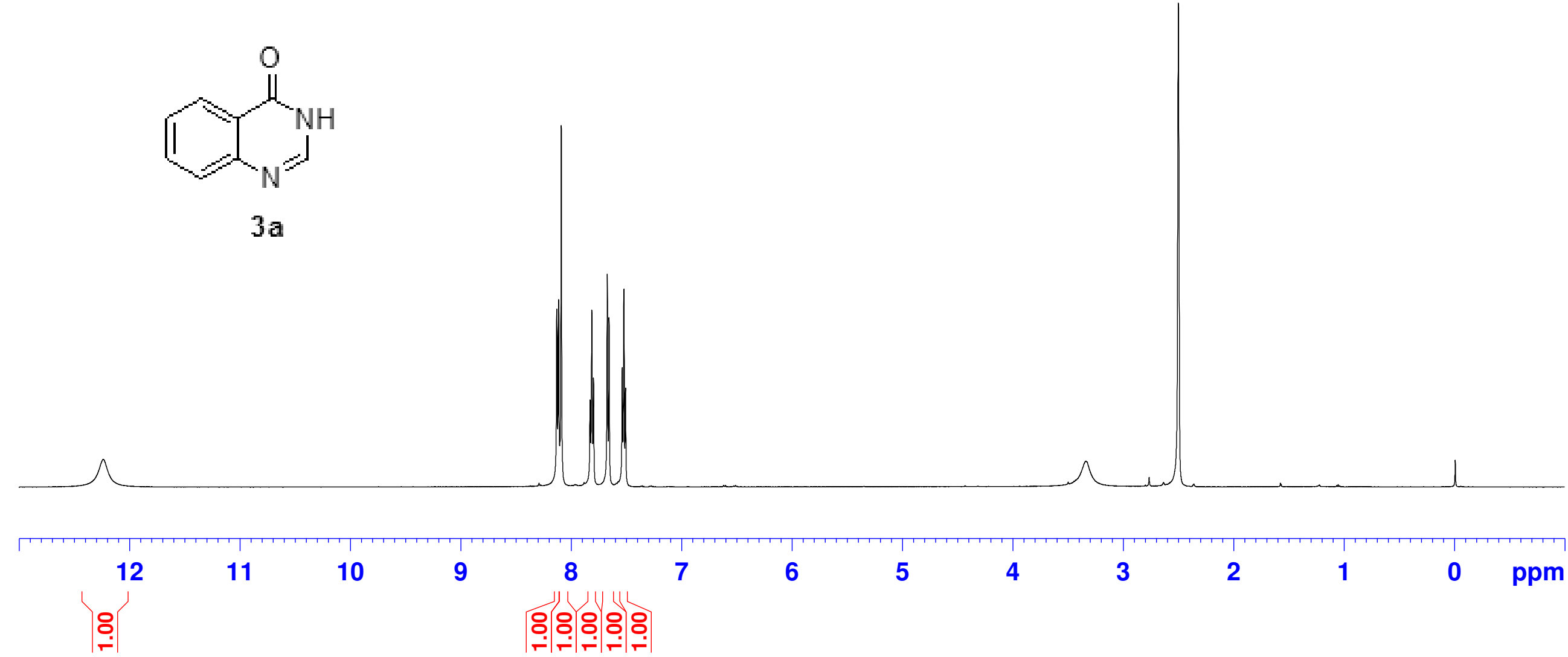

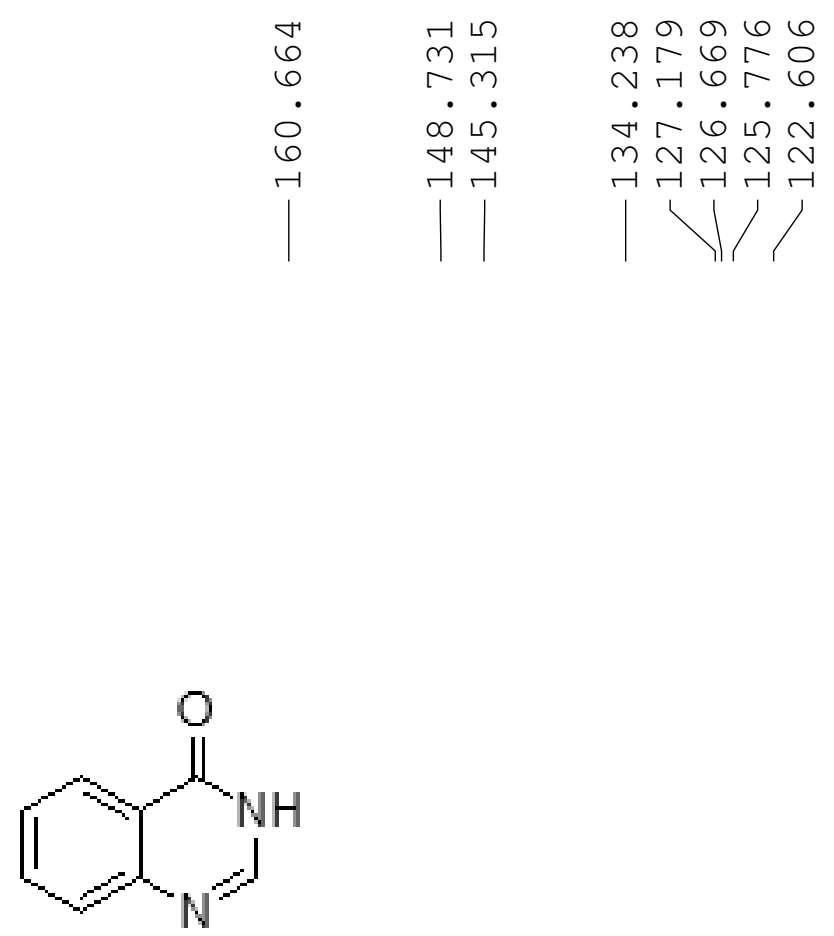

$3 a$

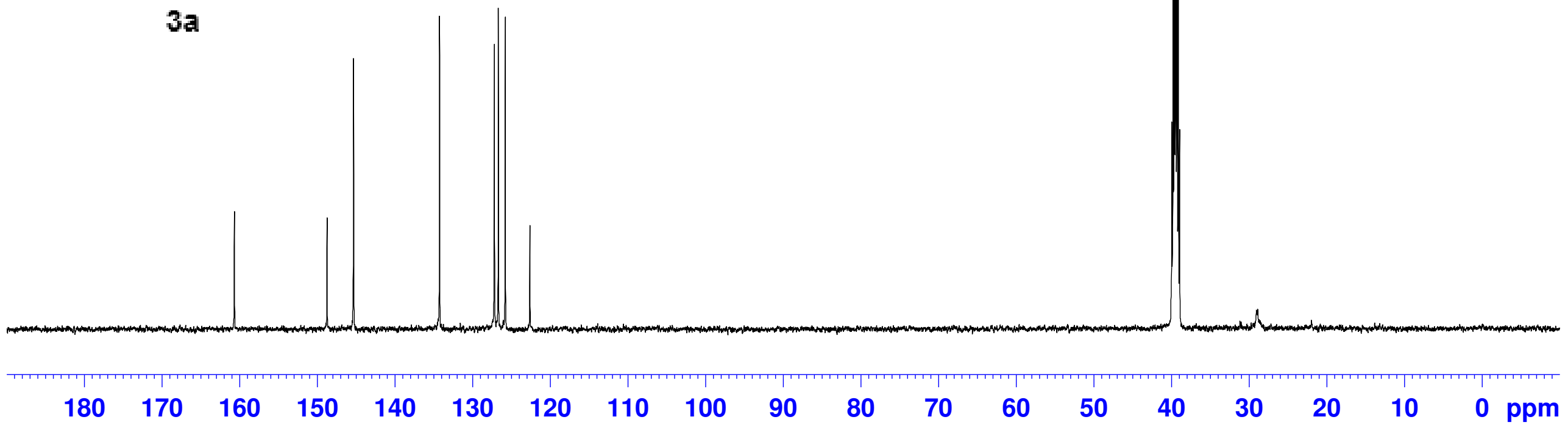



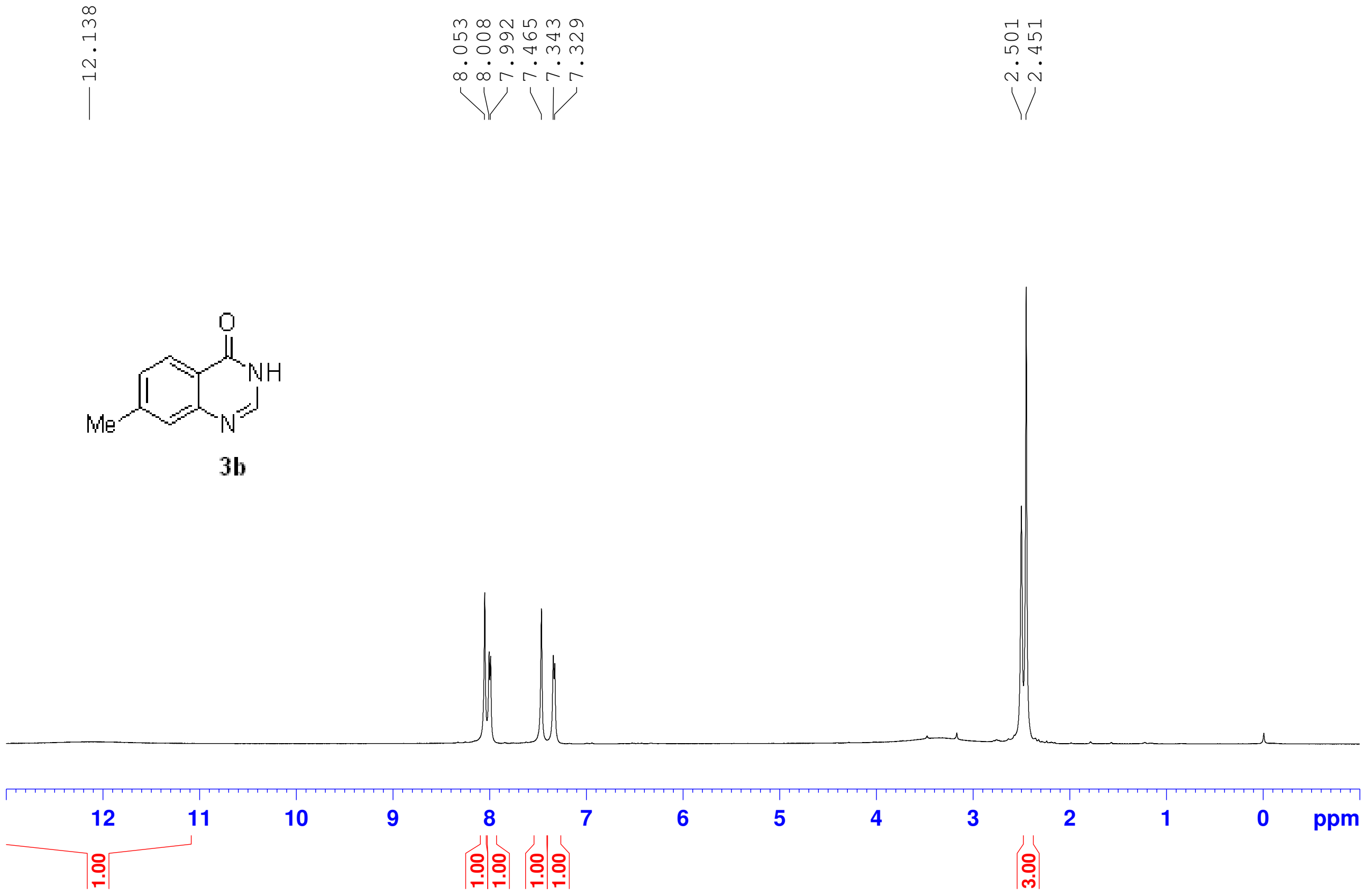


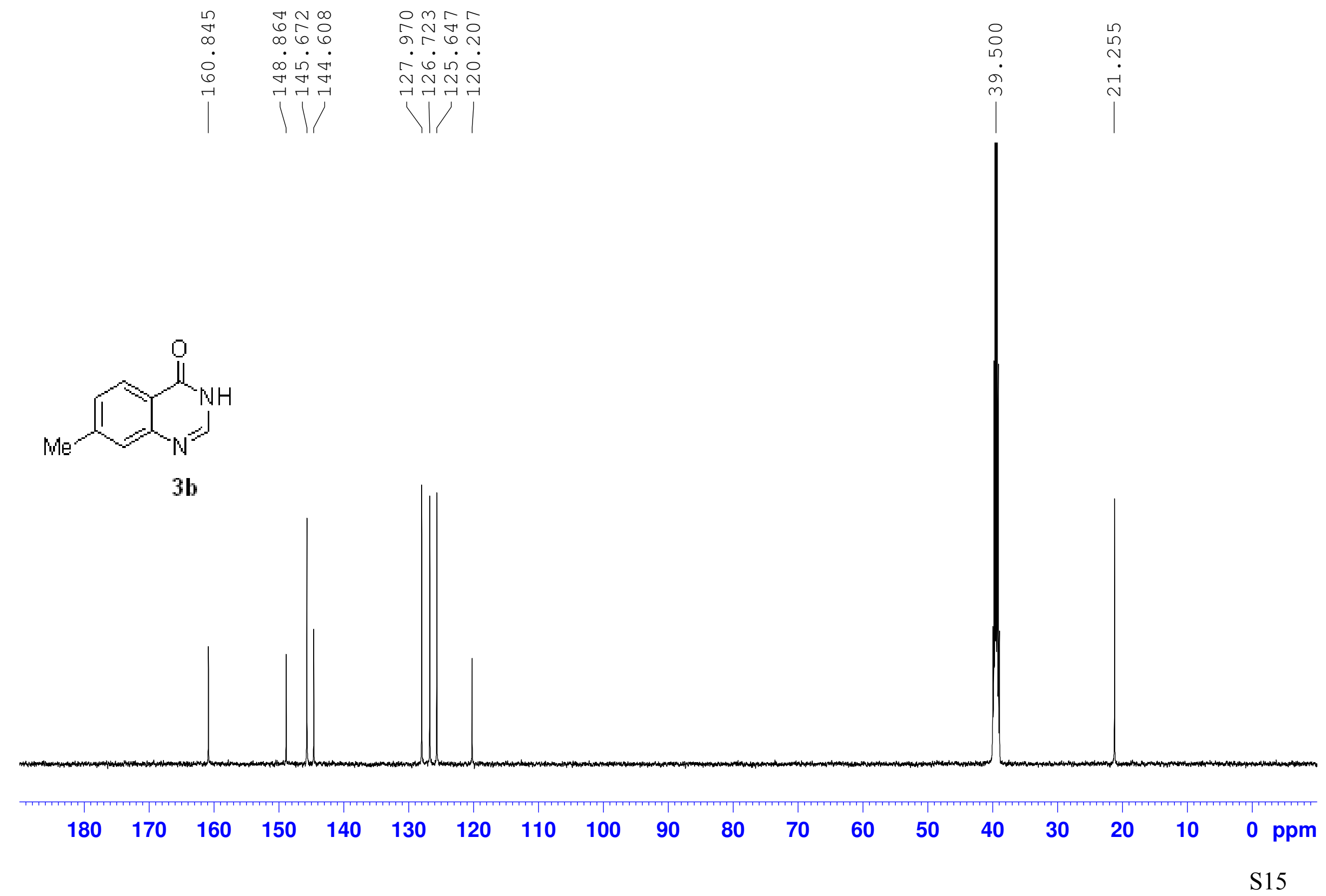




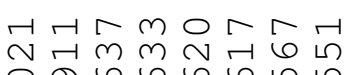

o.

0

1 $\circ \stackrel{n}{\circ}$

$\sin \frac{1}{4}$

$\dot{\sim} \dot{\sim}$

1<smiles>Cc1ccc2nc[nH]c(=O)c2c1</smiles>
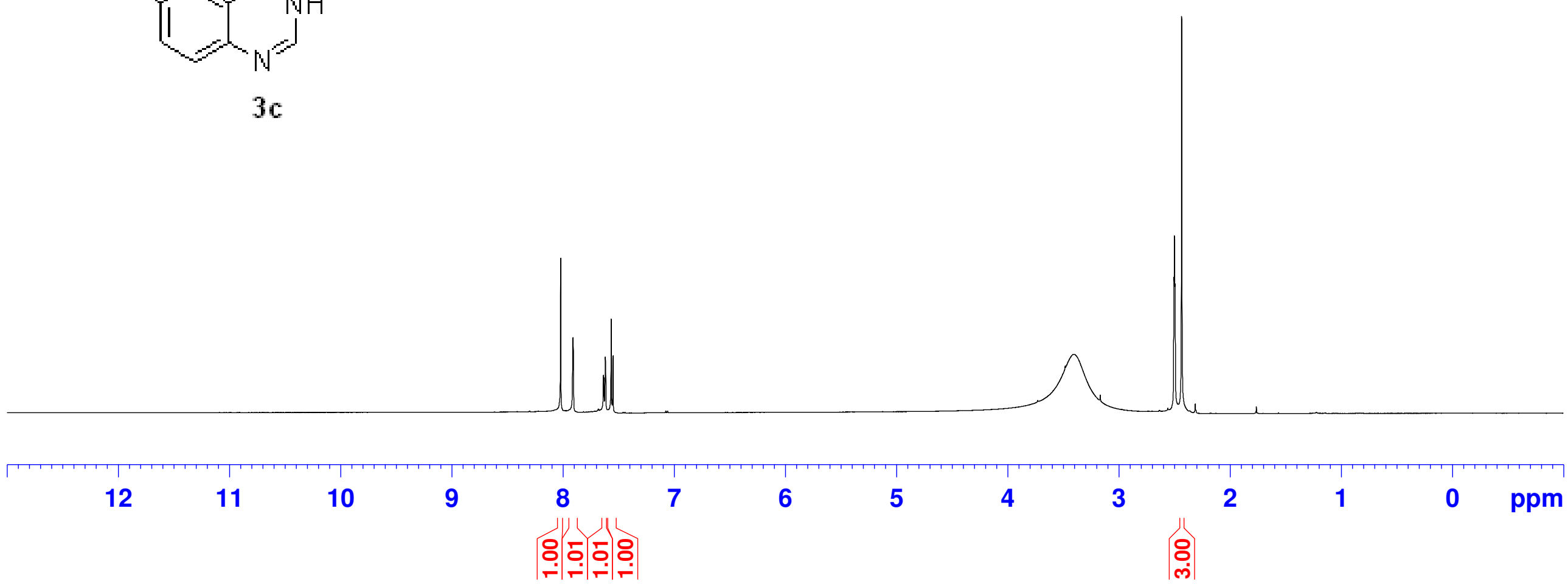

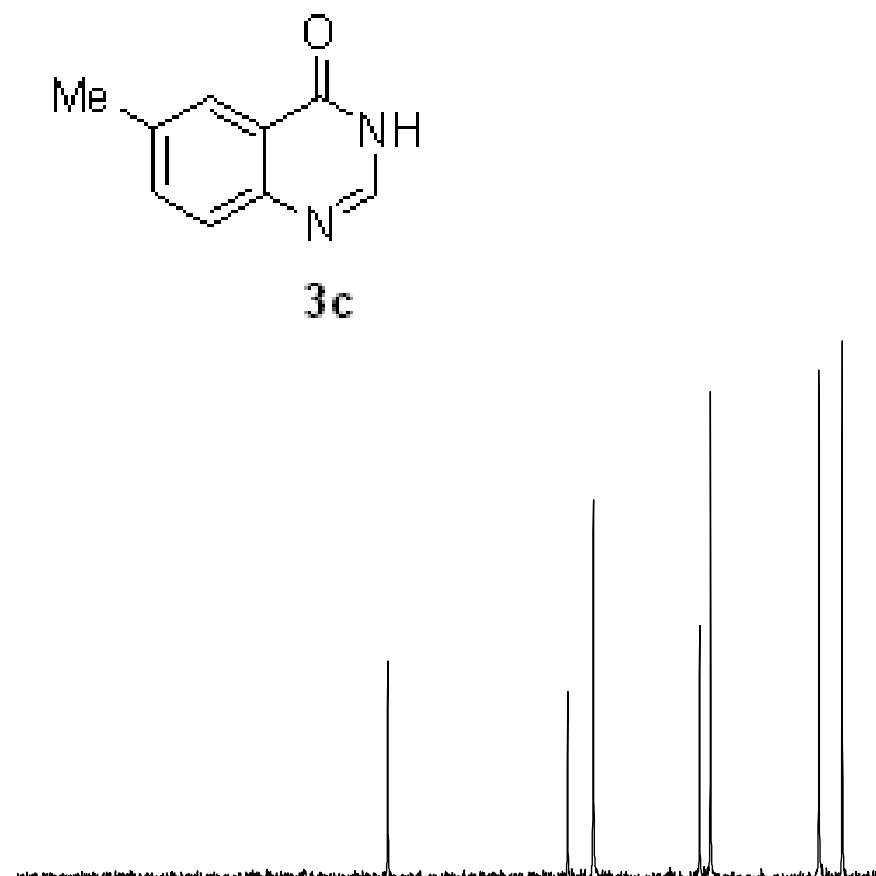

180

170160

150

$140 \quad 130$

120

110100

90

80

70

60

50

40

30

20

10

0 ppm 
Hハ

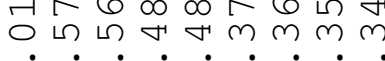

$\dot{0} \dot{0} \dot{0} \cdot \dot{r}$

$\longrightarrow$
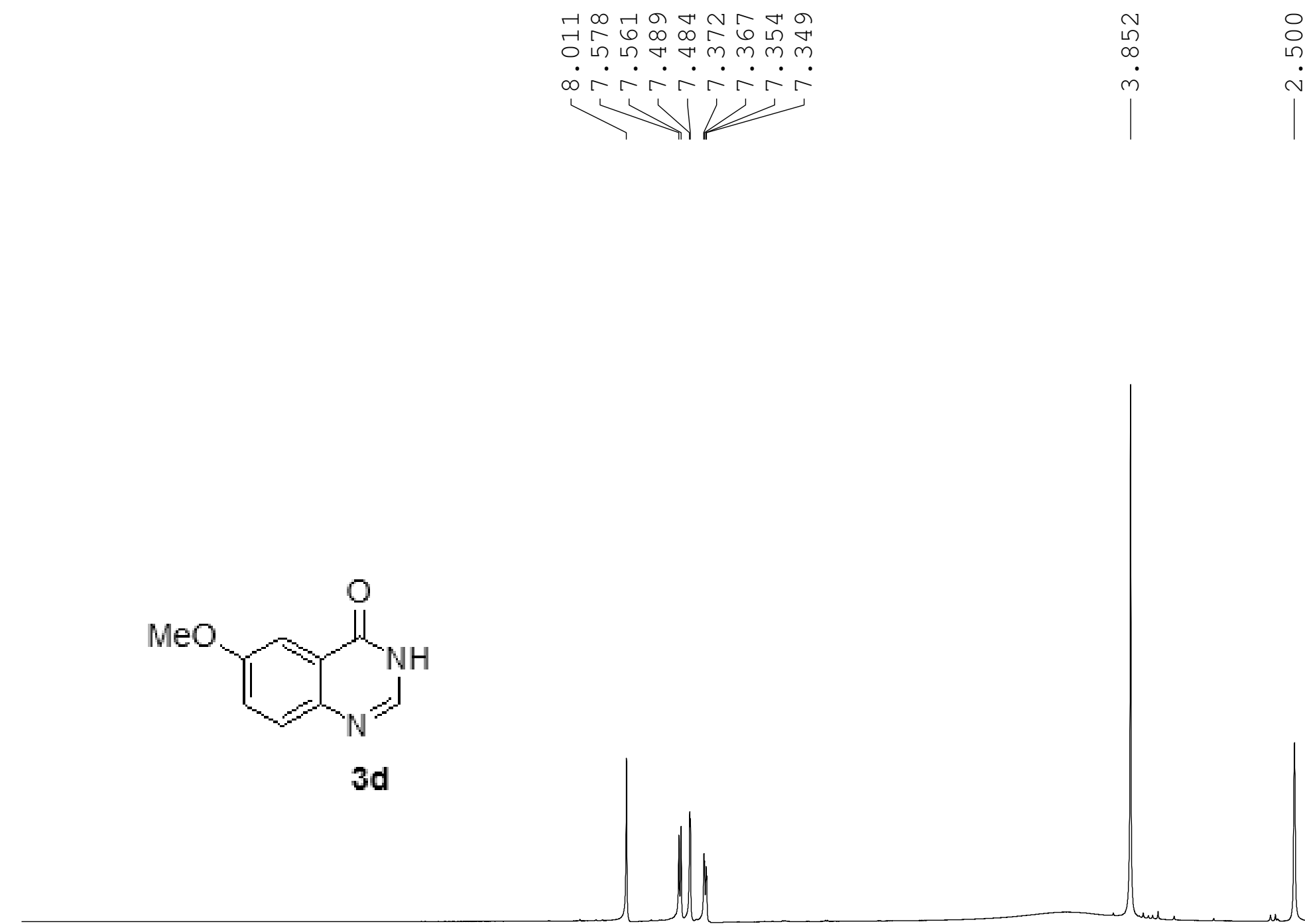

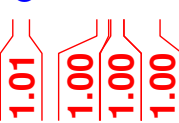

7

6

5

4

3

2

1

0 ppm

(항 

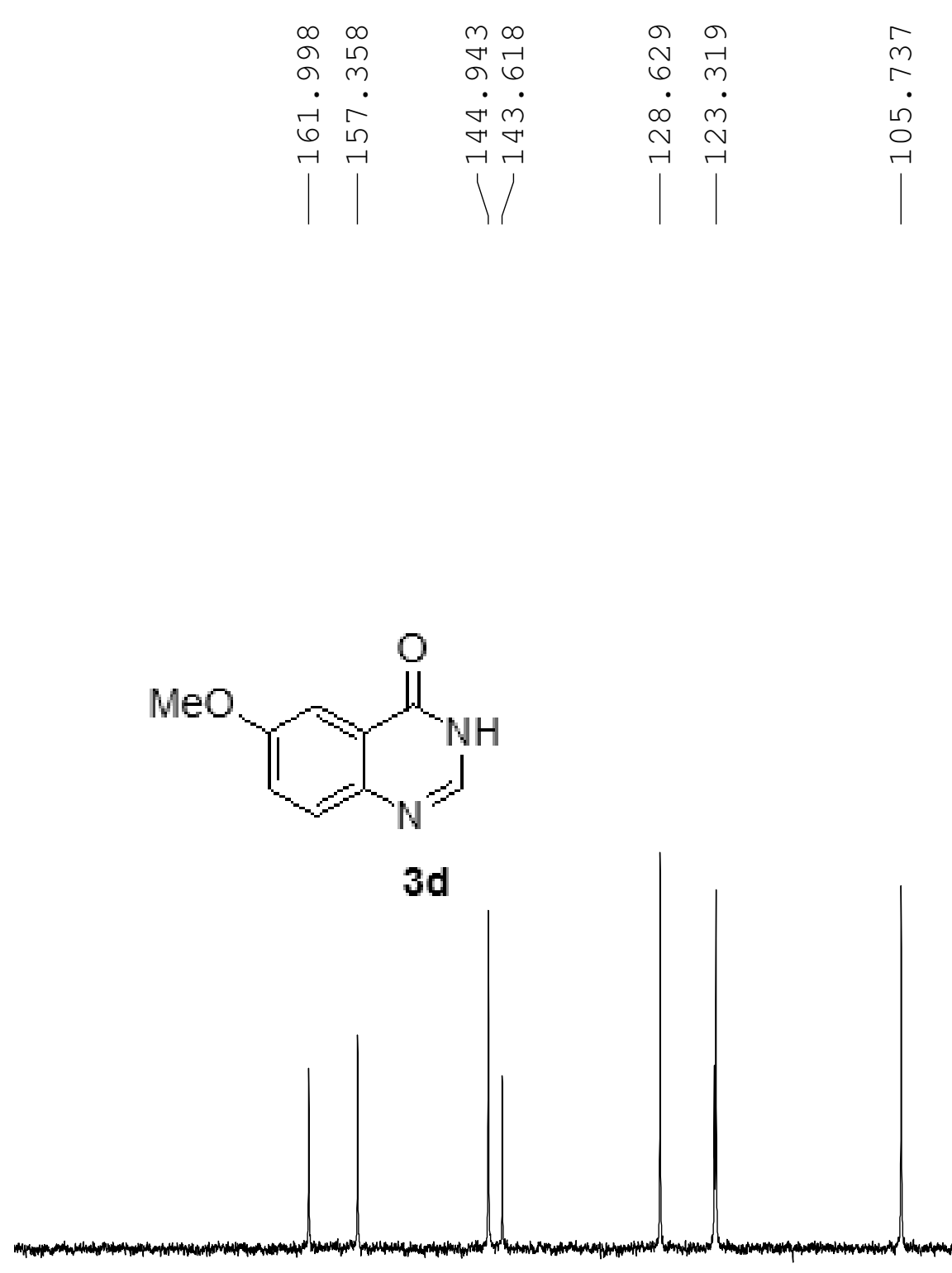

180

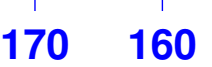

150

$140 \quad 130$

120

110100

90

80

$70 \quad 60$

50

40

30

20

10

0 ppm 
6,7-dimethoxyquinazolin-4 (3H)-one PROTON
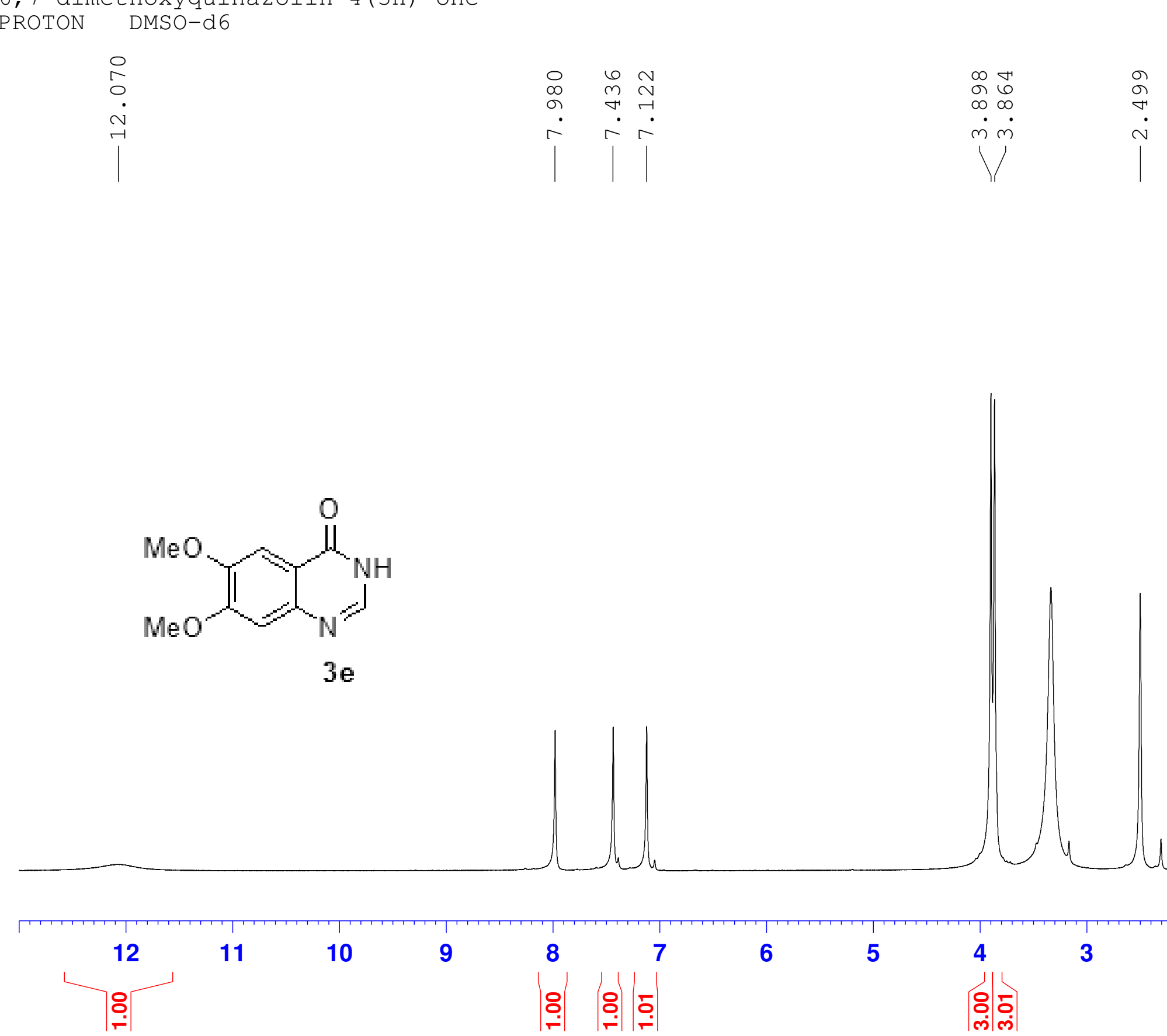

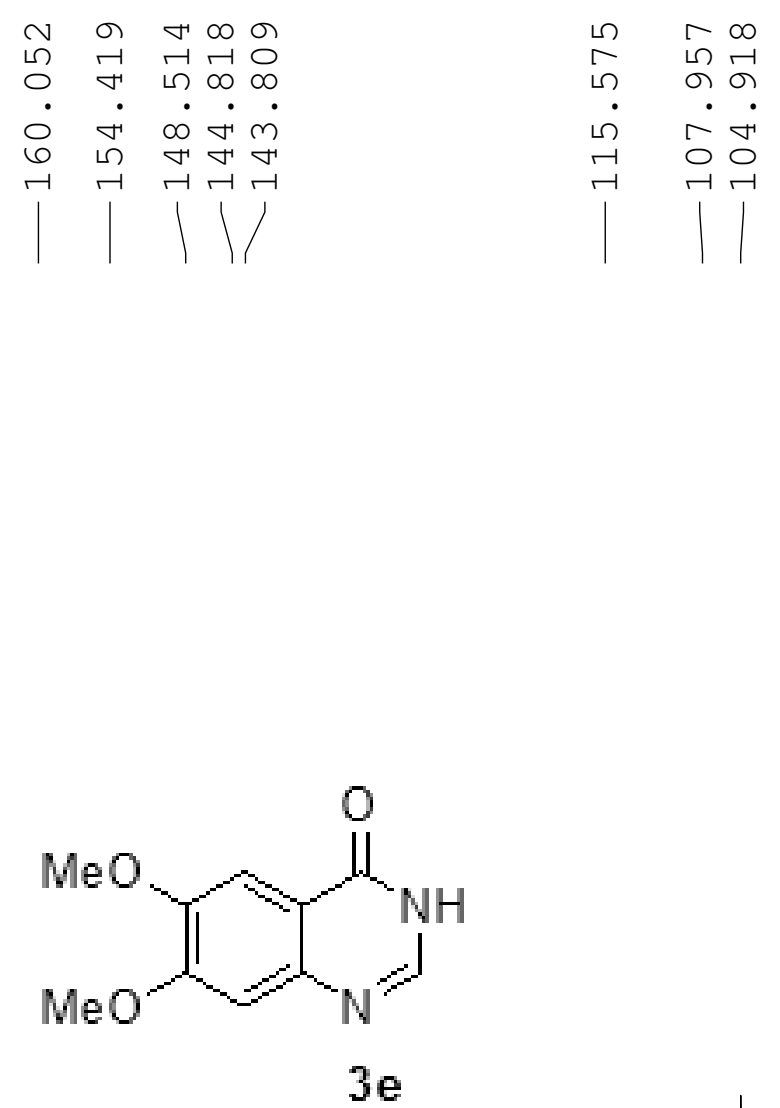


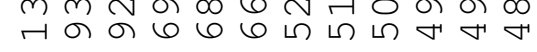
minririririn

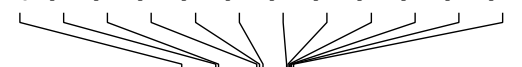<smiles>O=c1[nH]cnc2c(F)cccc12</smiles>

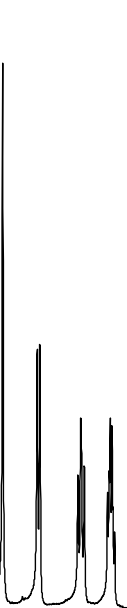

13

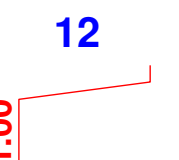

11

10

9

8

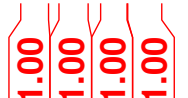



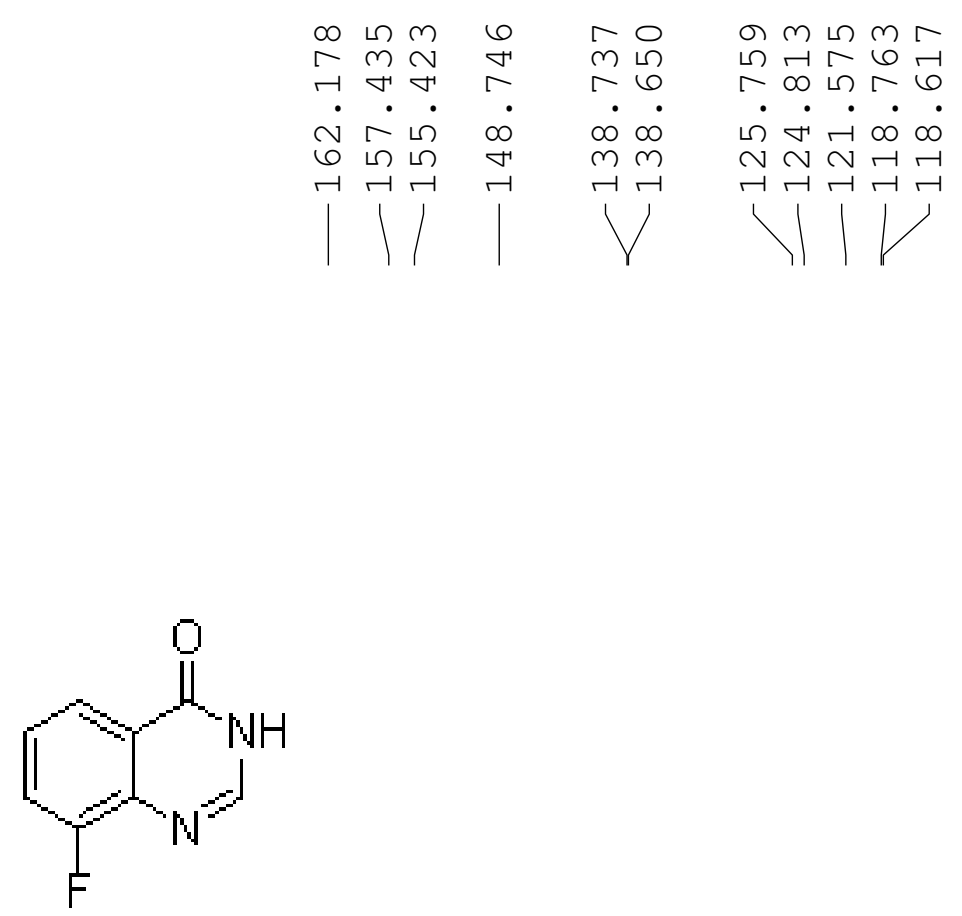

$3 f$
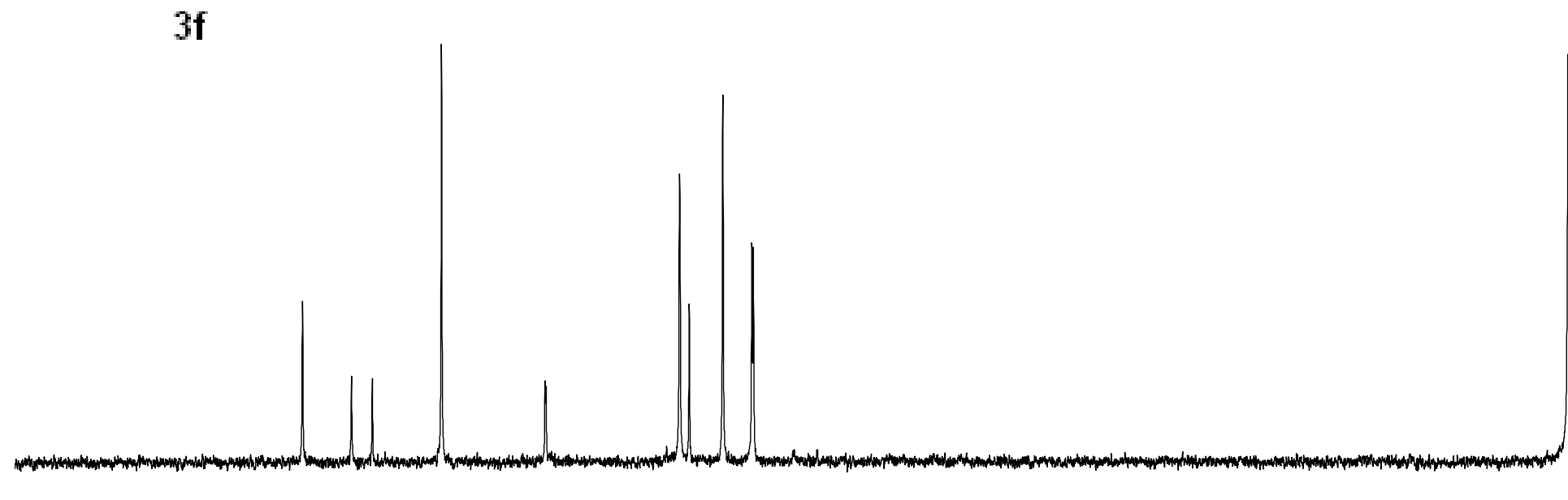

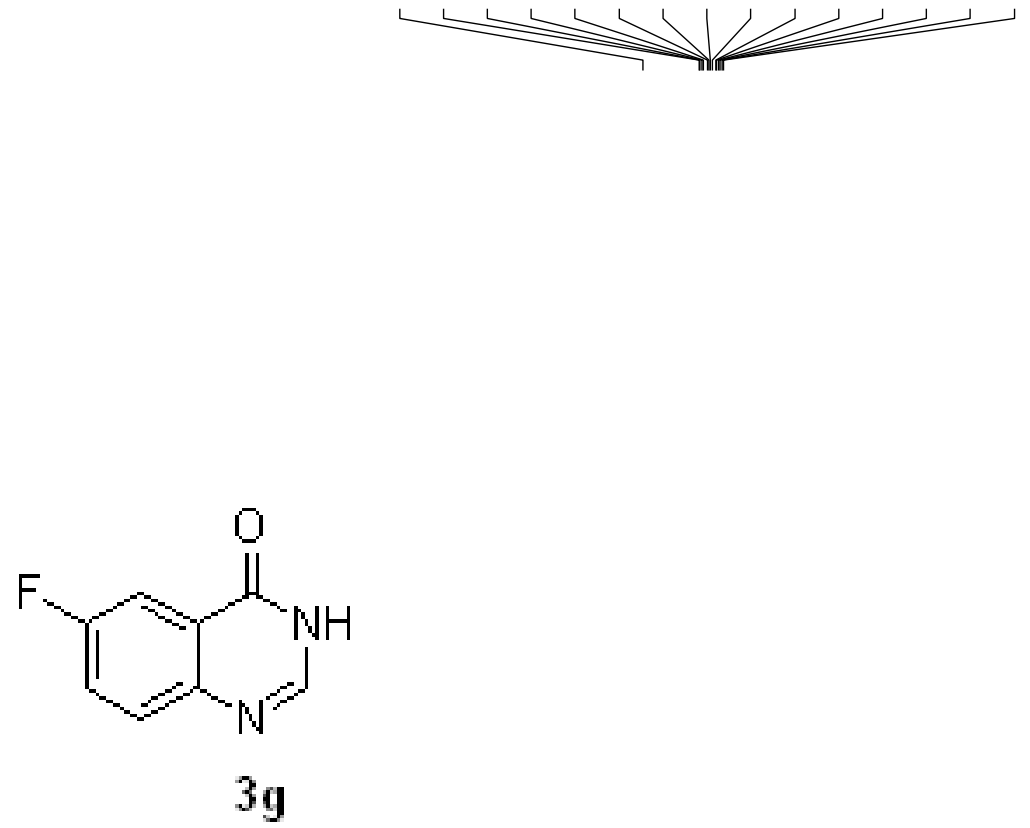


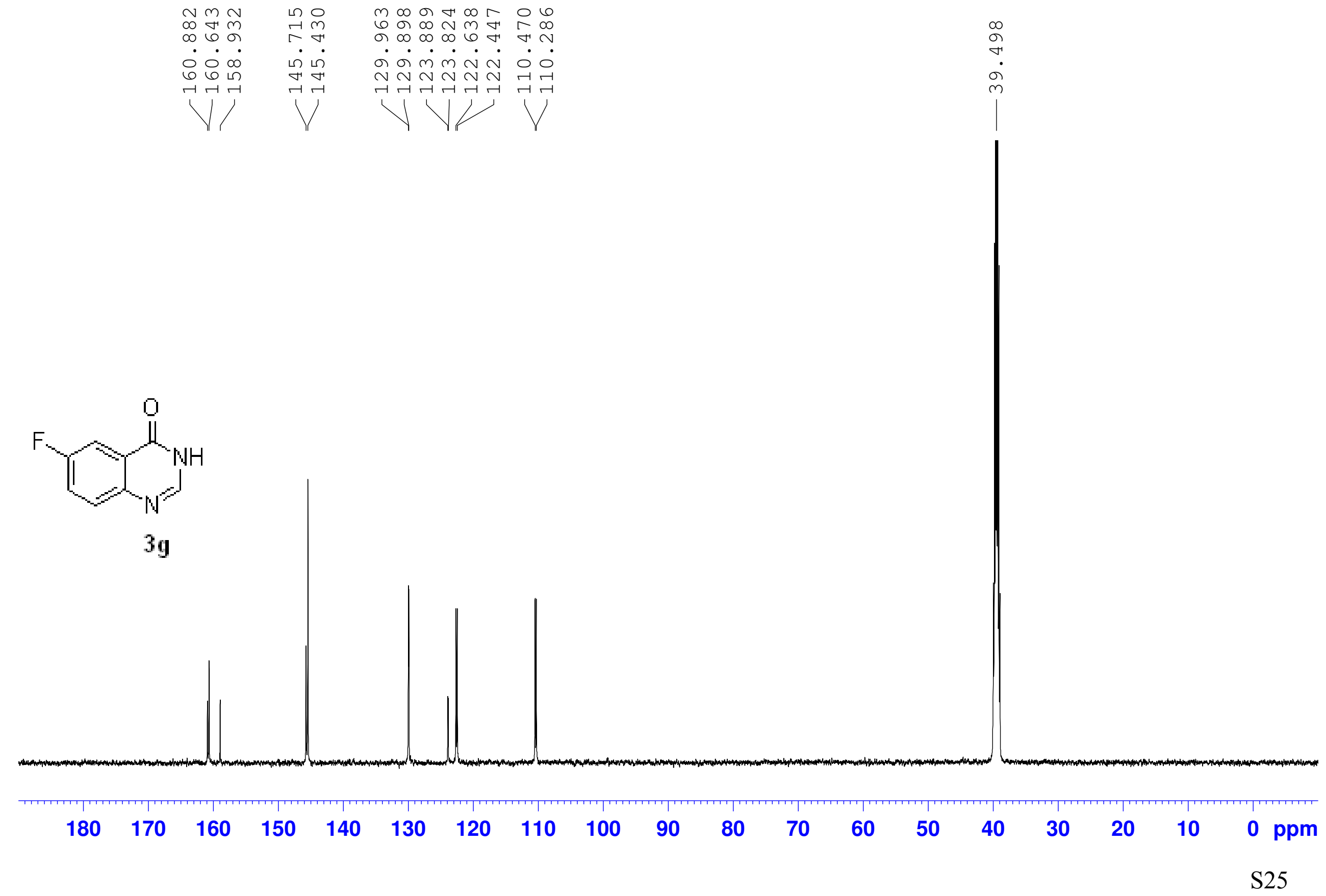


8-chloroquinazolin-4 (3H)-one

PROTON DMSO-d6
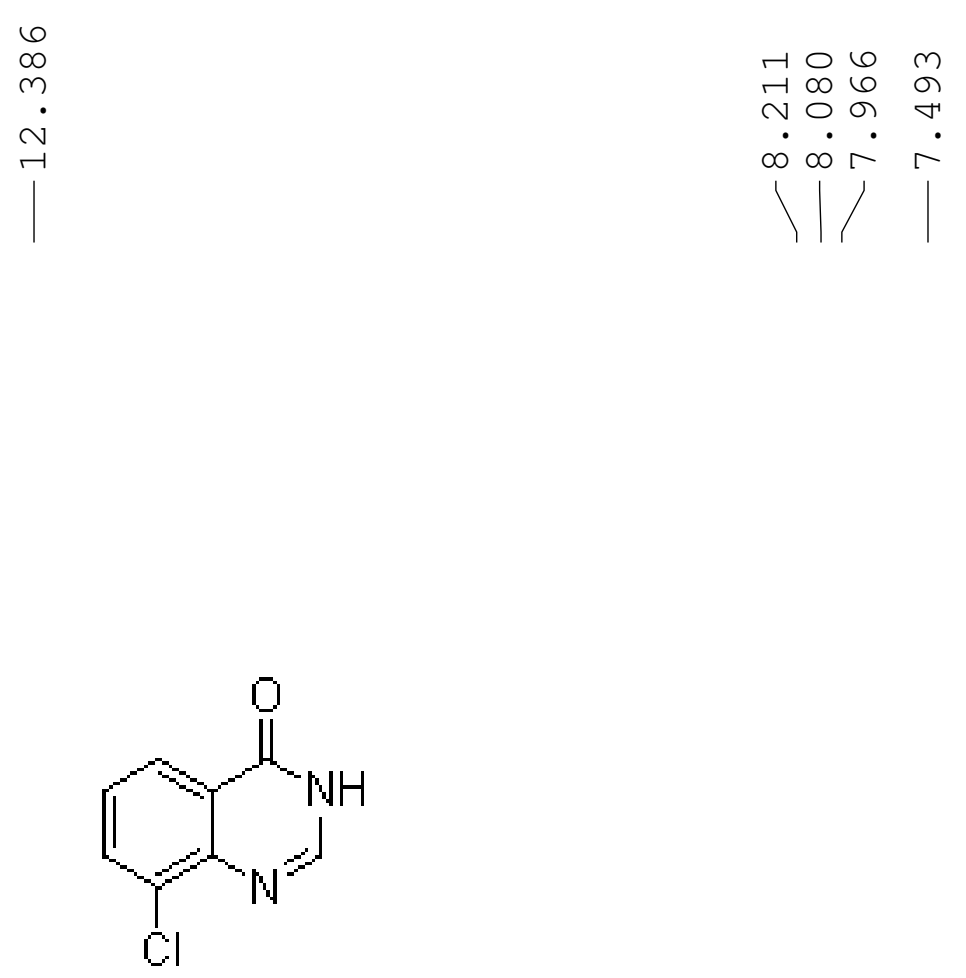

$3 \mathbf{h}$
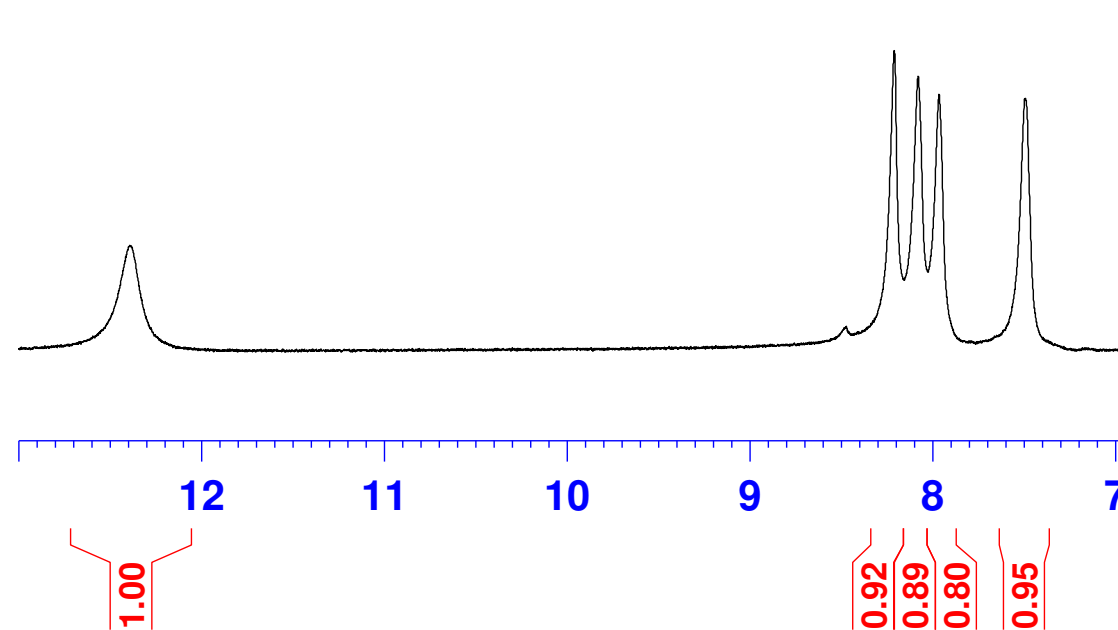

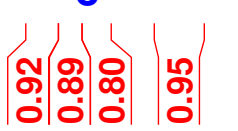




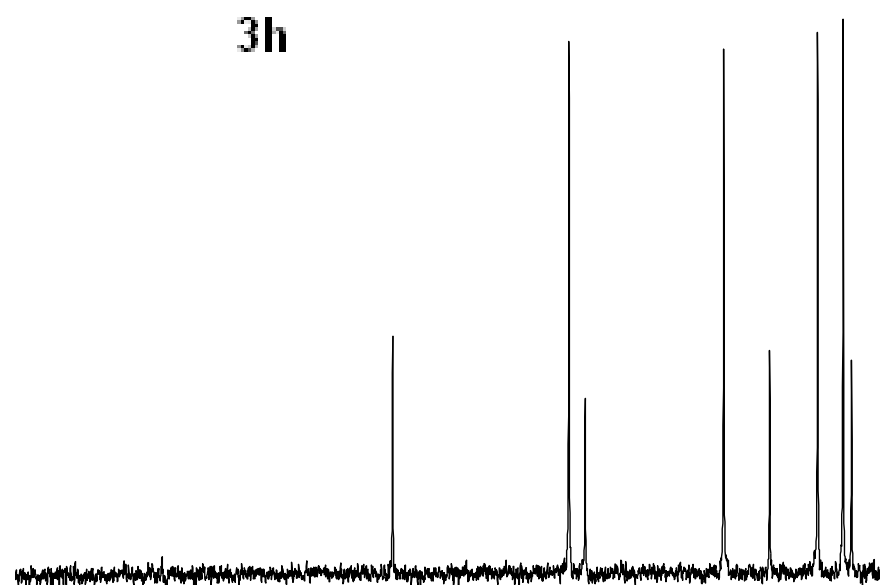


7-chloroquinazolin-4 (3H)-one

PROTON DMSO-d6
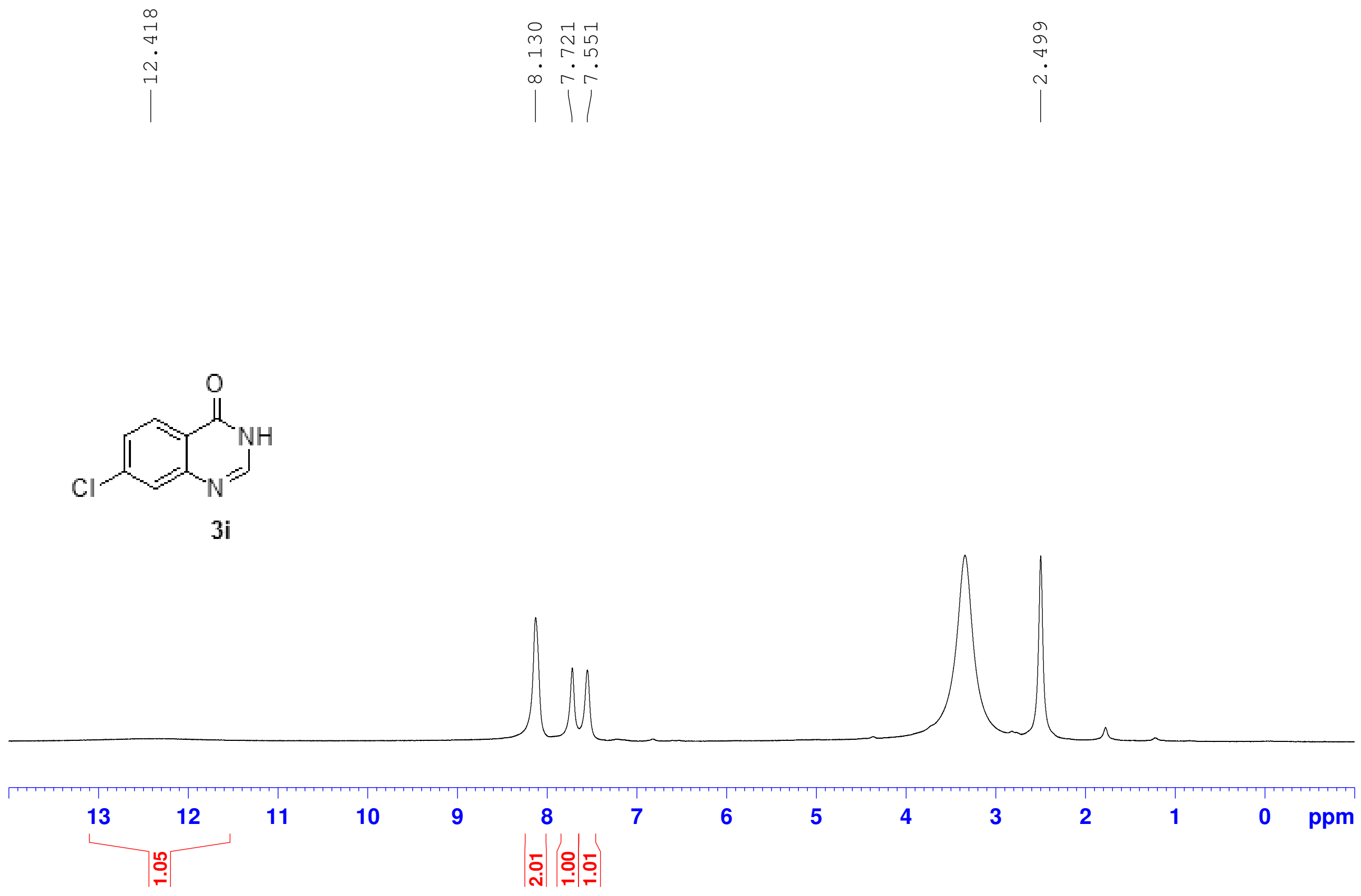
<smiles>O=c1[nH]cnc2cc(Cl)ccc12</smiles>

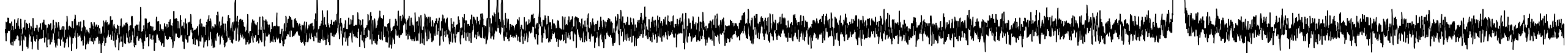




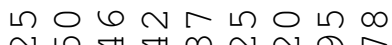

닥하

$\dot{0} \infty \dot{a} \dot{a} \dot{r} \dot{r}$

$\longrightarrow$<smiles>O=c1[nH]cnc2ccc(Cl)cc12</smiles>

$3 \mathbf{j}$ 


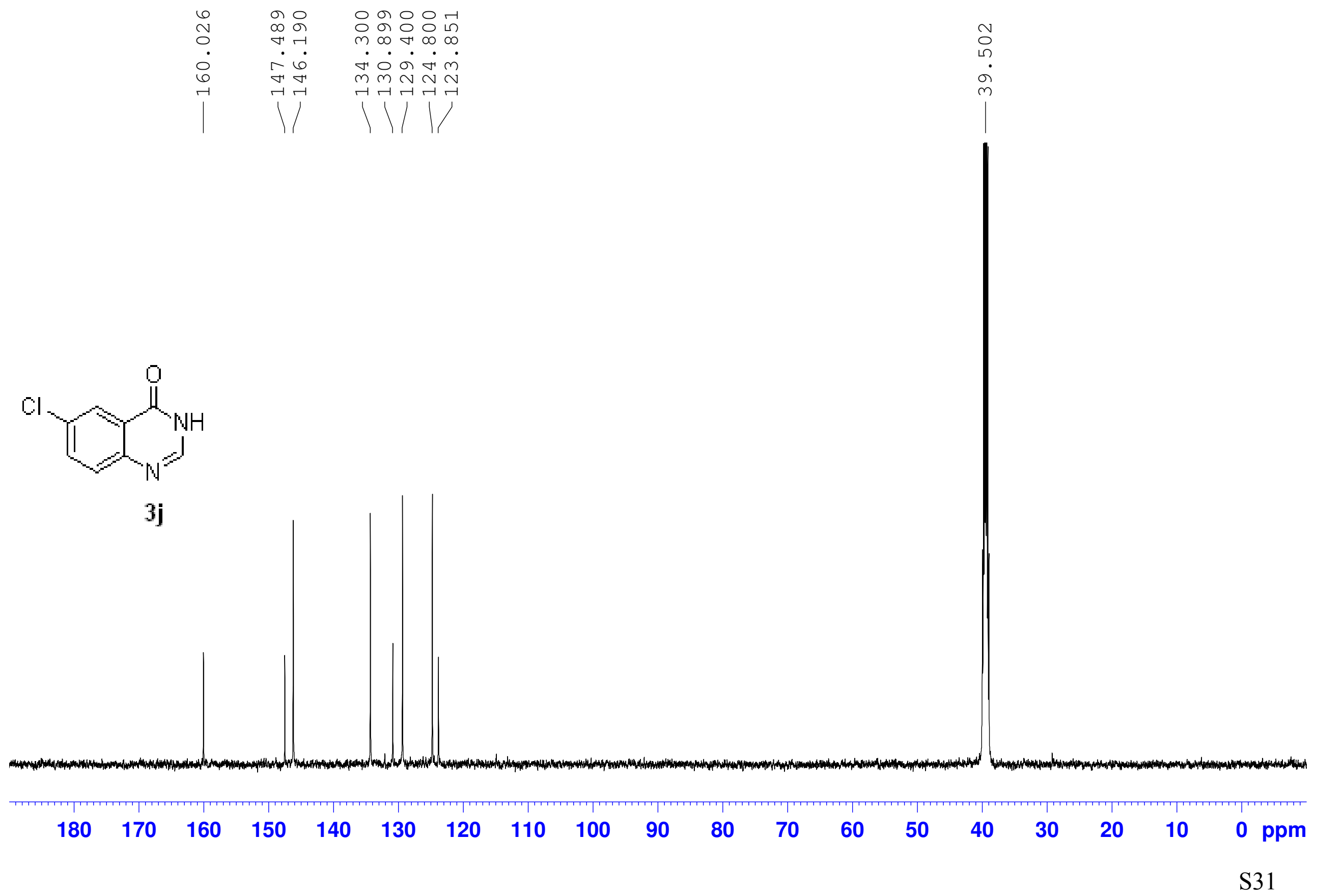




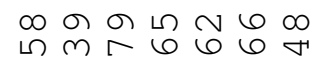

$\begin{array}{llllll}\pi & \infty & \infty & \infty & \infty & 0\end{array}$

$\dot{\infty} \dot{\infty} \dot{0} \dot{\sim} \dot{\sim}$<smiles>O=c1[nH]cnc2cc(Br)ccc12</smiles>

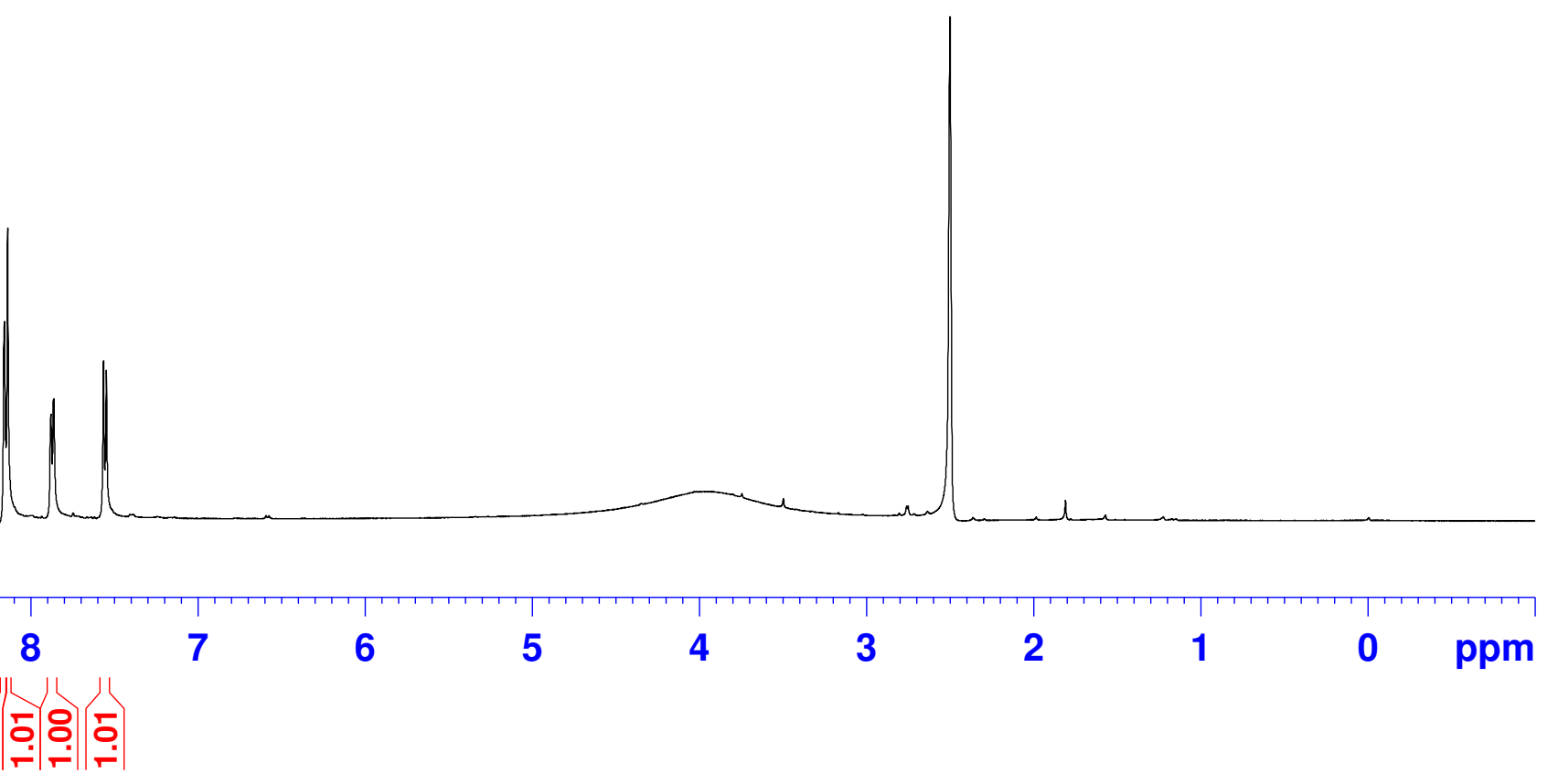


<smiles>O=c1[nH]cnc2cc(Br)ccc12</smiles>

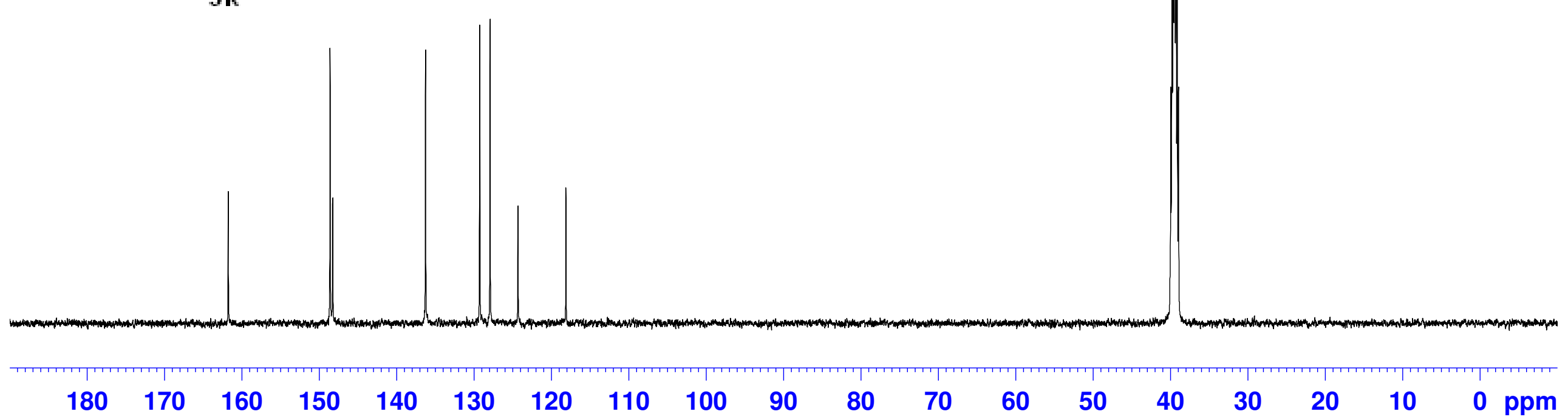


6-bromoquinazolin-4 (3H)-one

PROTON DMSO-d6

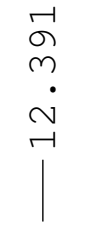

ऽ)

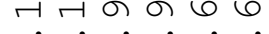

$\infty \dot{r} \dot{2} \dot{0}$

MV

ㅇํㅇ

$\dot{\sim}$

$\sim$<smiles>O=c1[nH]cnc2ccc(Br)cc12</smiles>
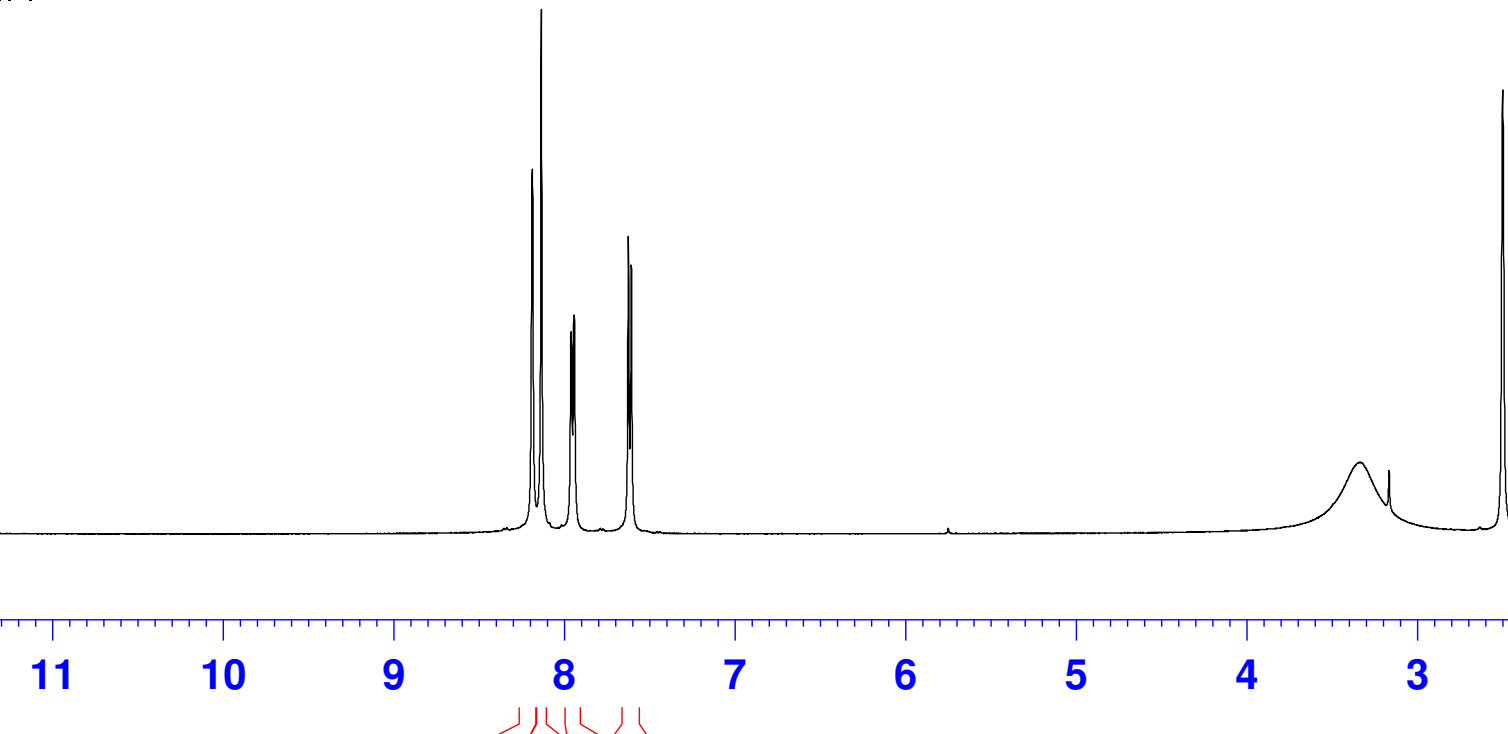

9

8

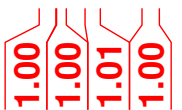

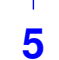

4

3

2

1

0 ppm

움 

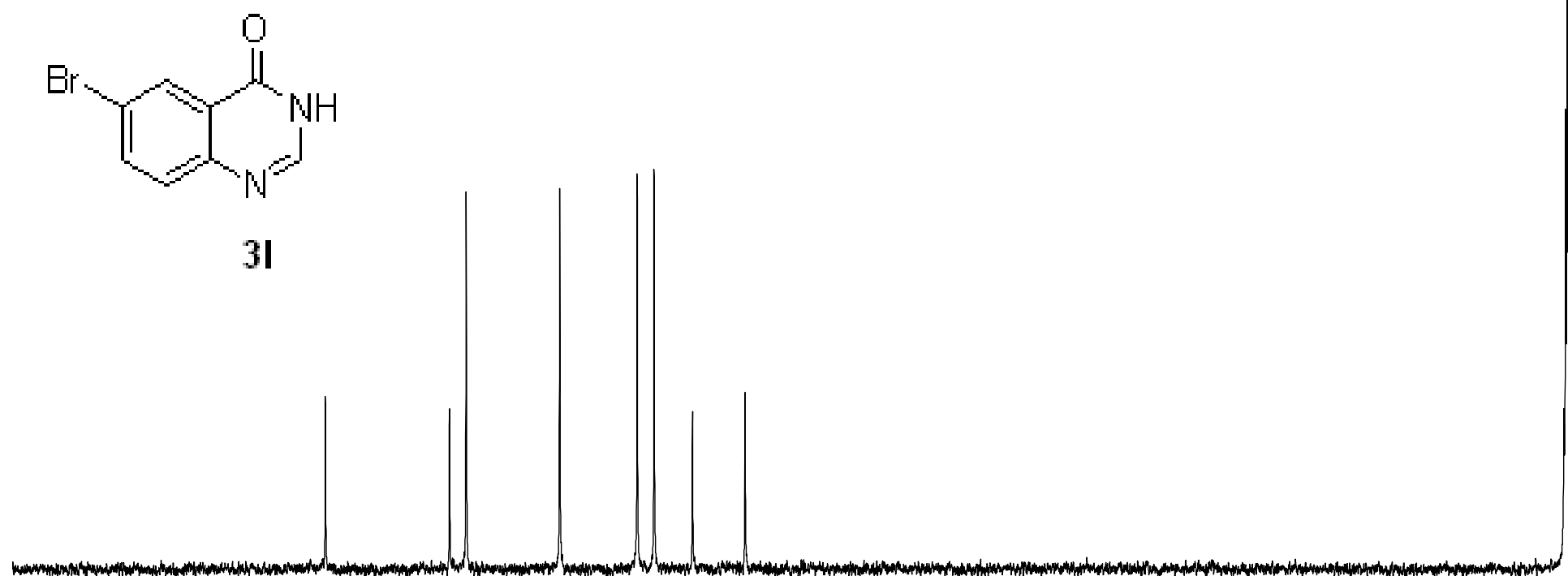
7-(trifluoromethyl) quinazolin-4 (3H) -one PROTON DMSO-d6

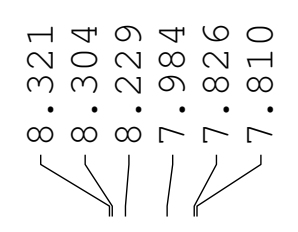

눙
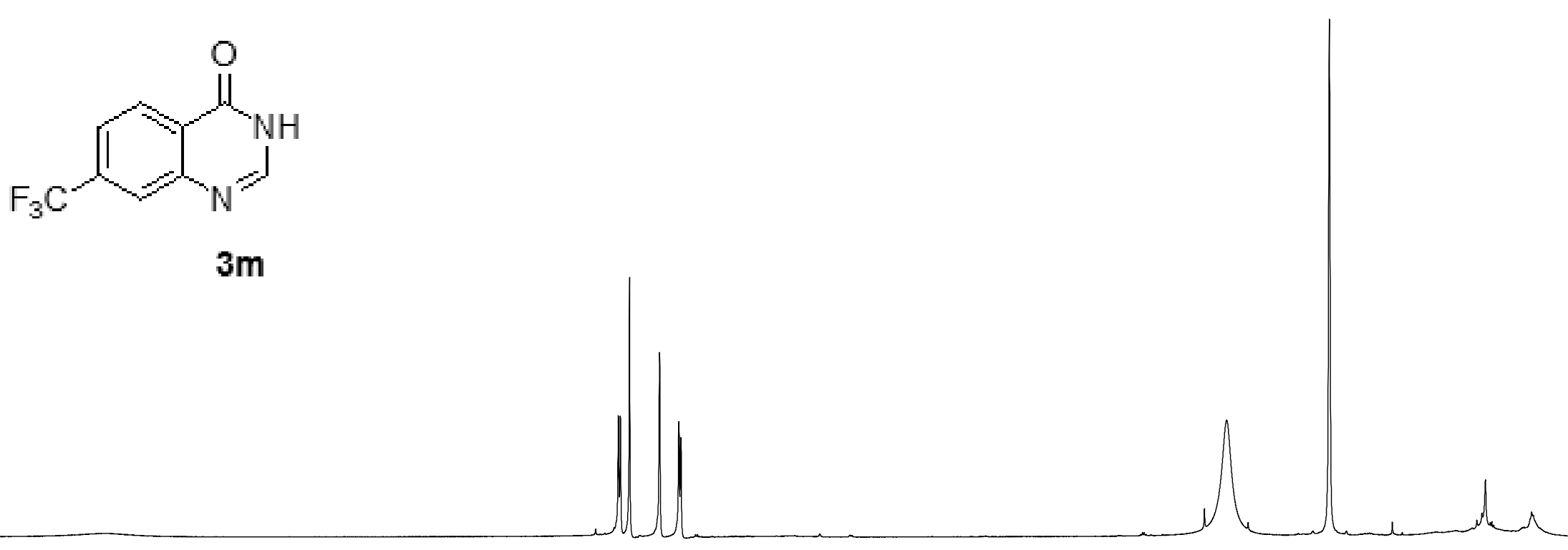

13

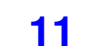

10 용.

8

7

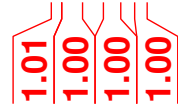




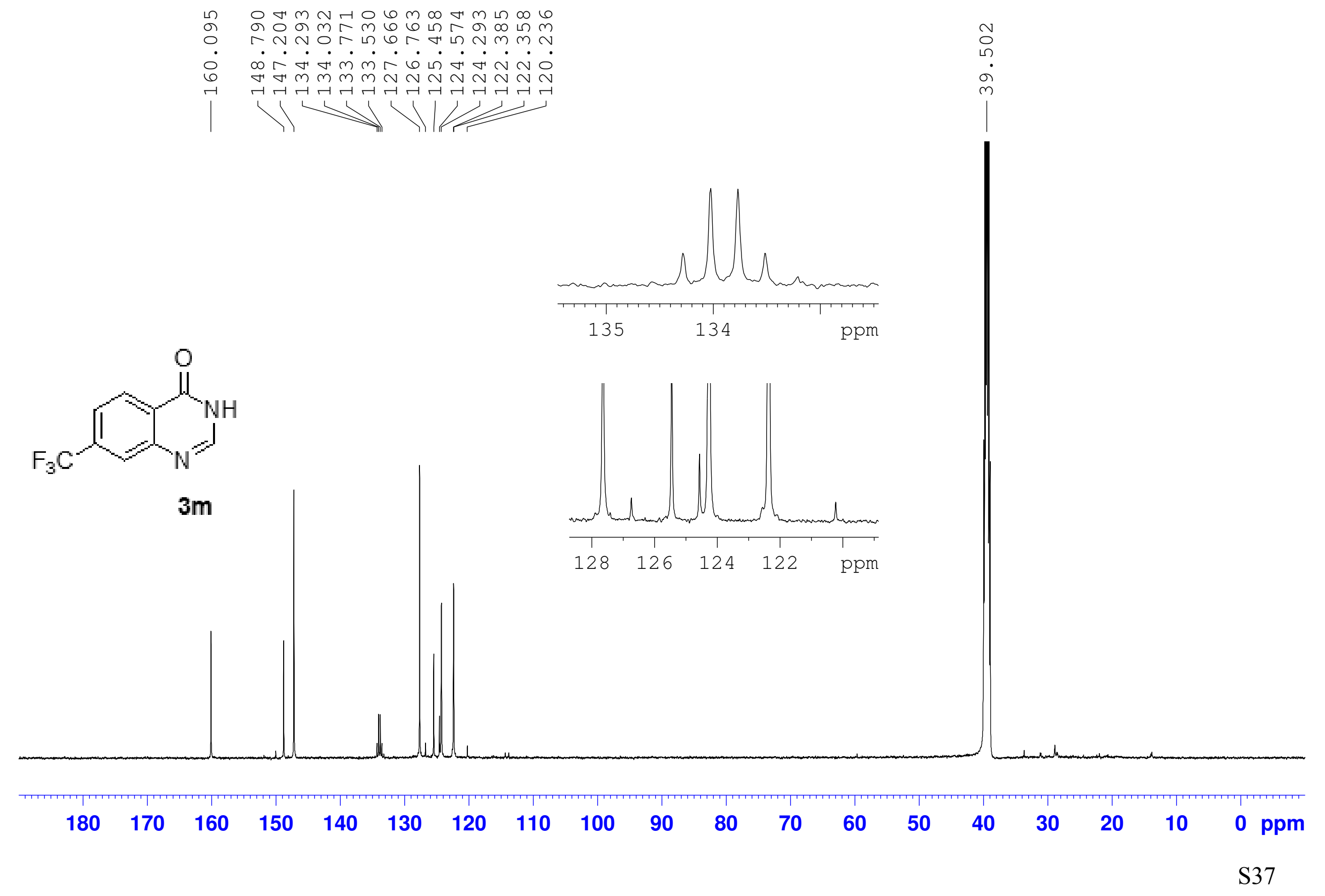


$\infty m+4 r v \in \circ m \infty r 0$

m

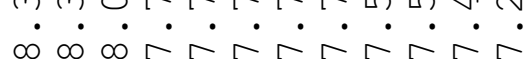

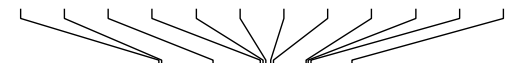

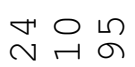

000

नंभi

II $\infty m$

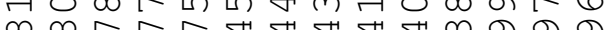

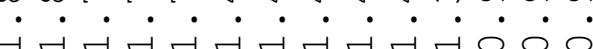

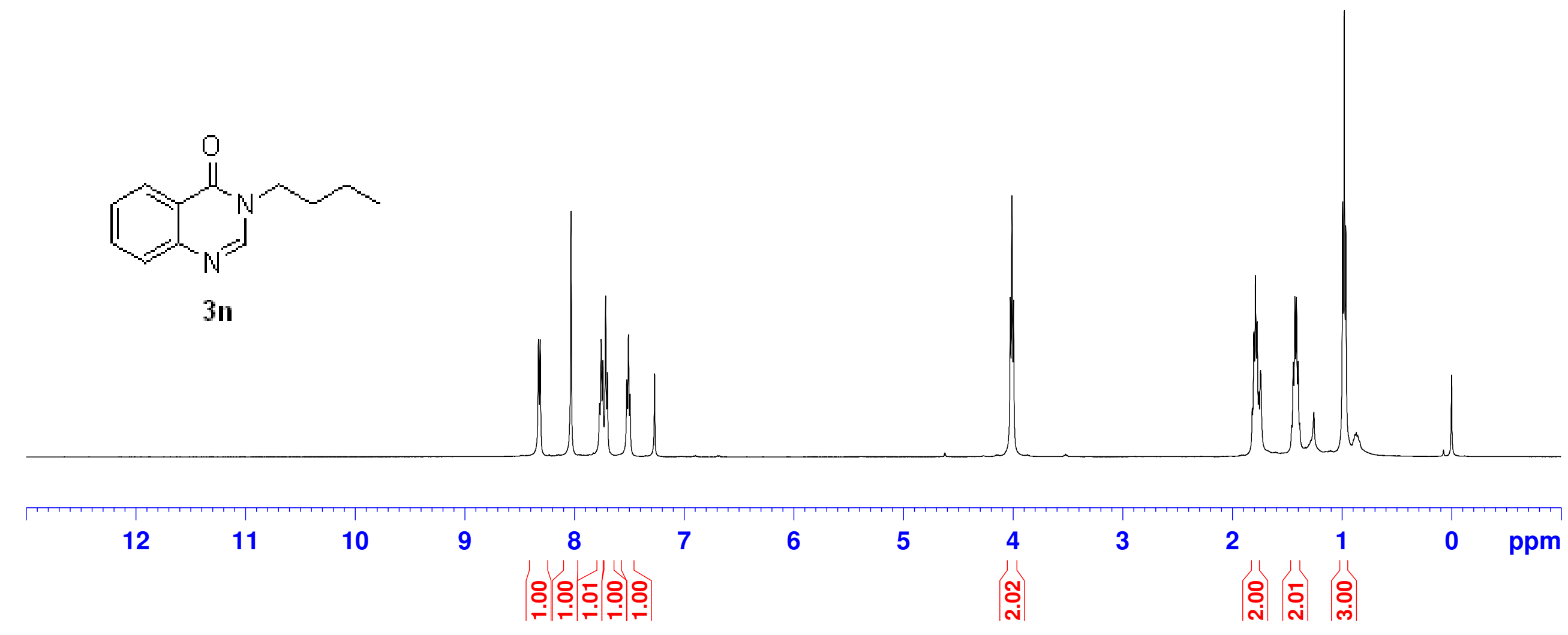




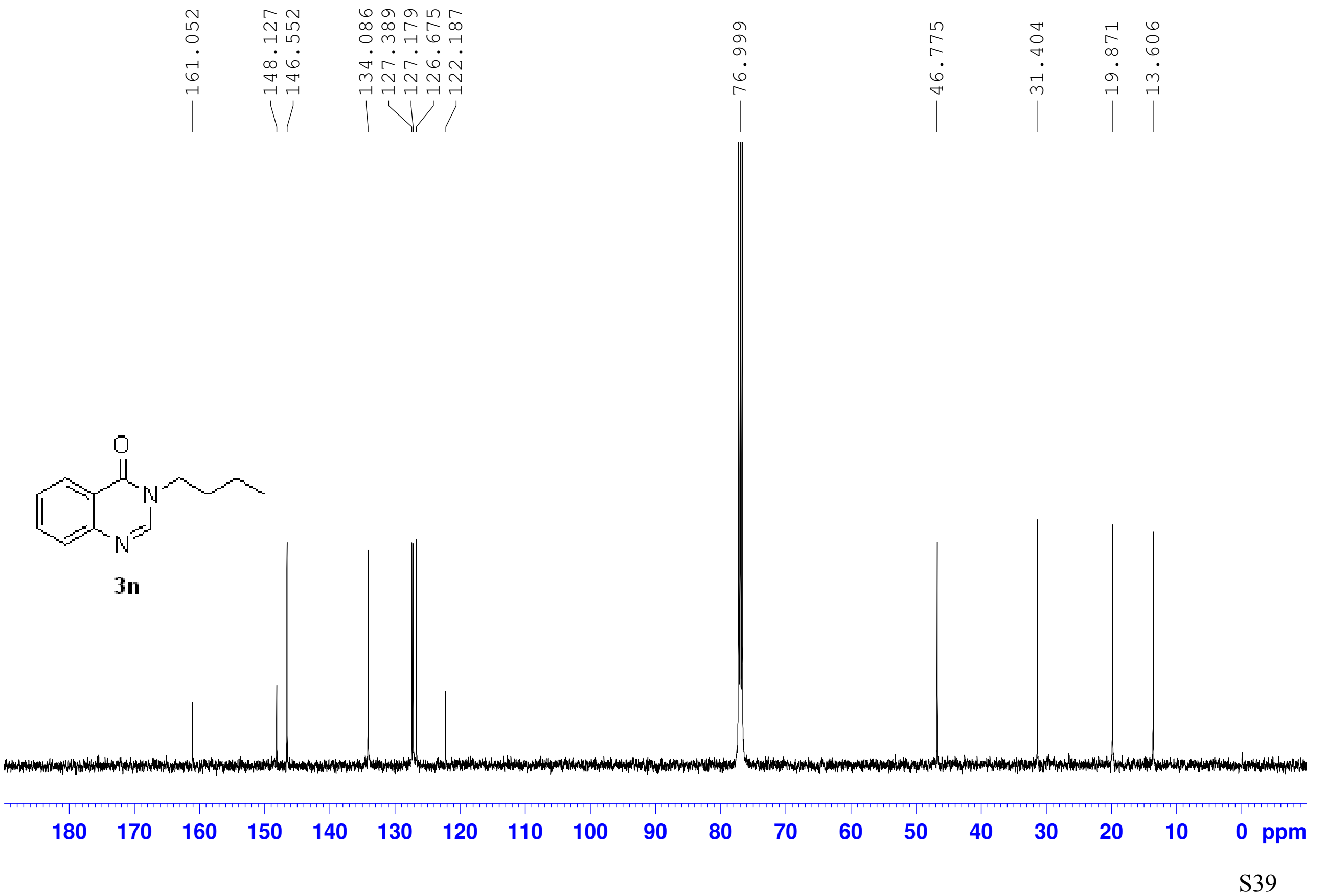


2H-benzo-1,2,4-thiadiazine 1,1-dioxide PROTON $\mathrm{DMSO}-\mathrm{d} 6$

$$
\begin{gathered}
\infty \\
m \\
\sim \\
\sim \\
+
\end{gathered}
$$
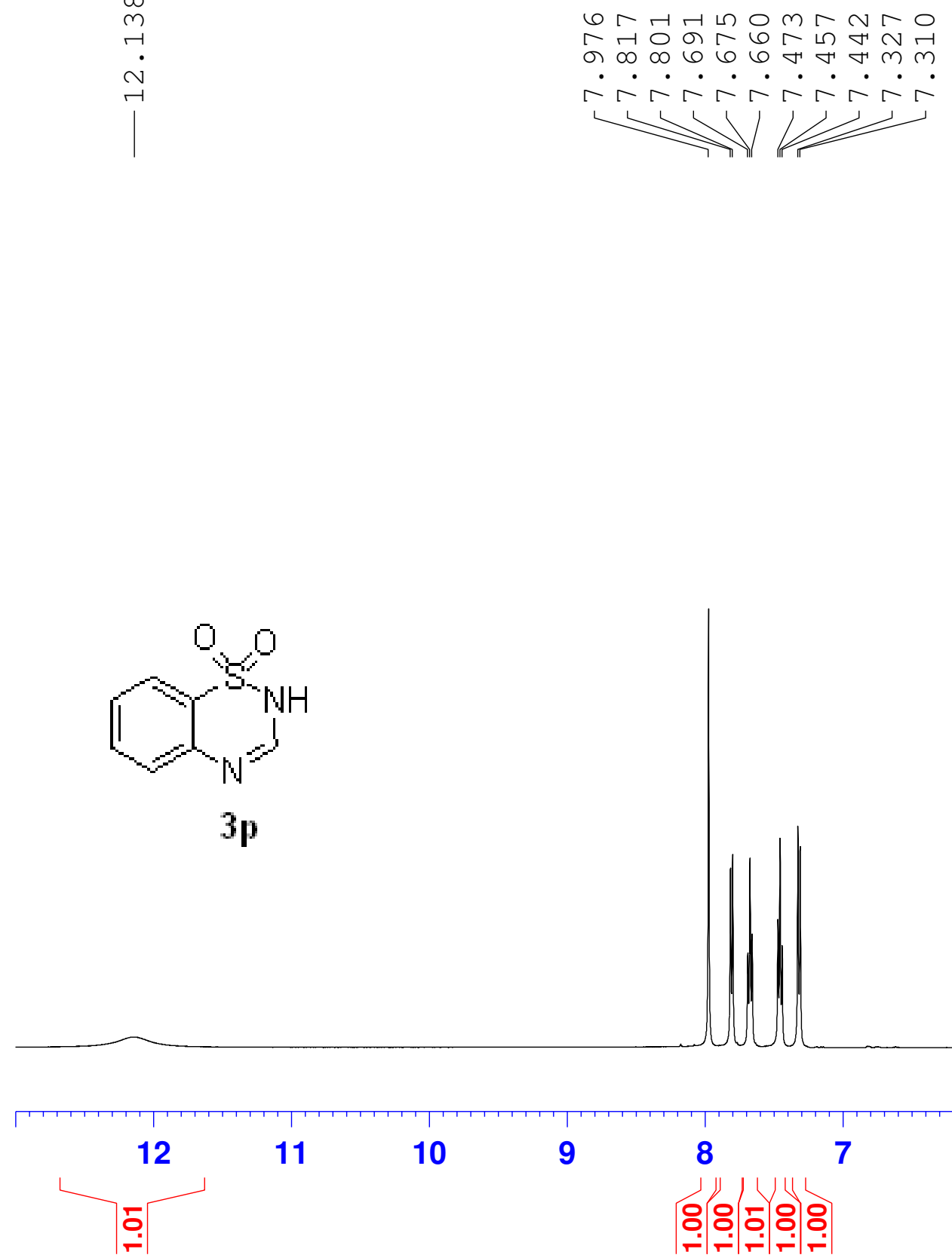

\section{8

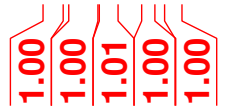



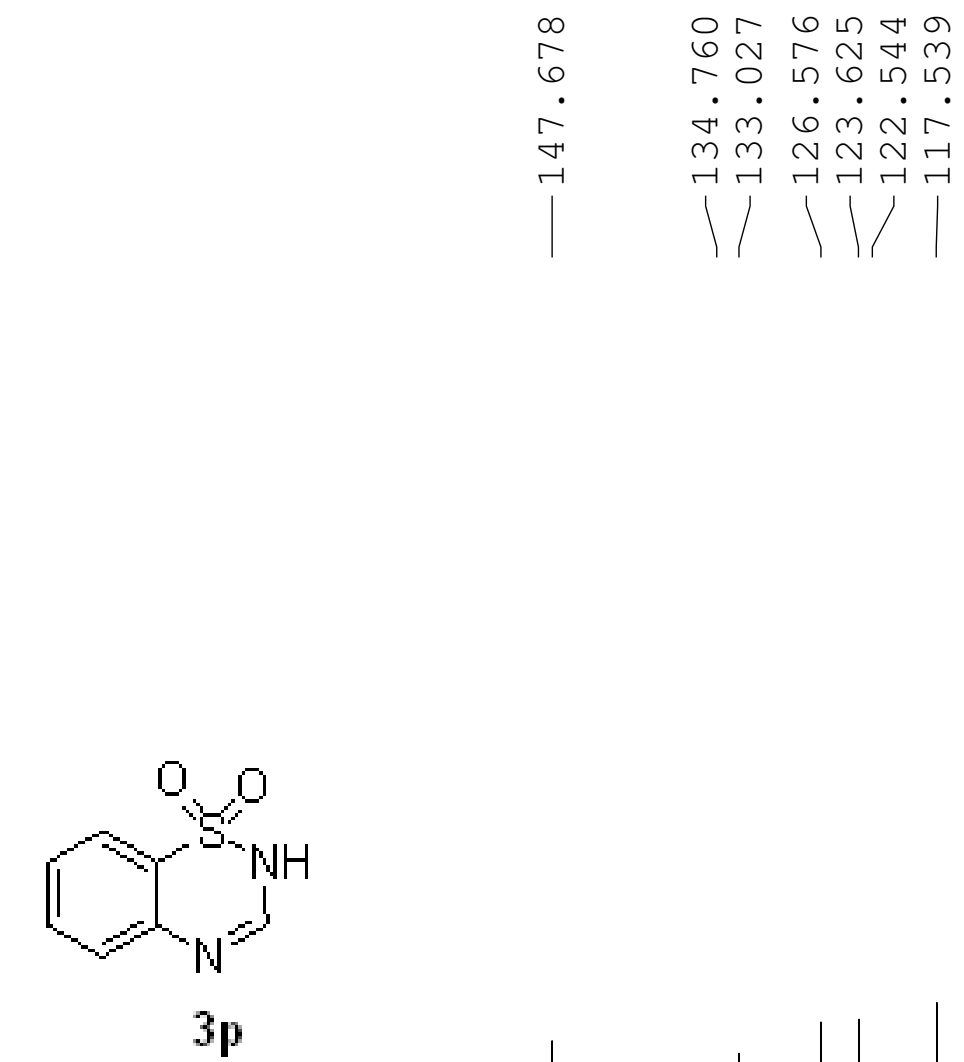

180

$170 \quad 160$

$40 \quad 130$

120

110100

90

80

70

60

50

40

30

20

10

0 ppm 
6,7-bis (2-methoxyethoxy) quinazolin-4 (3H)-one PROTON DMSO-d6

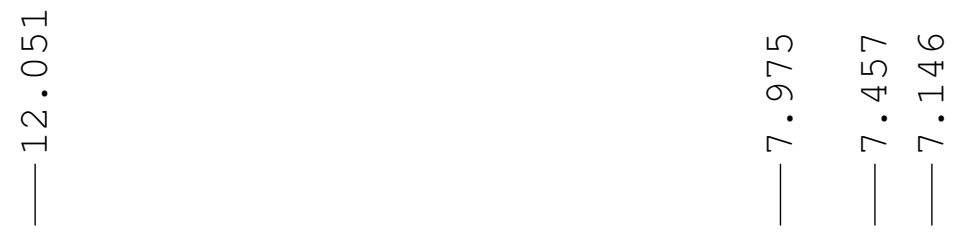

เூ

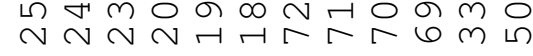
$\dot{\sigma} \dot{\gamma} \dot{\gamma} \dot{\sigma} \dot{\gamma} \dot{m} \dot{m} \dot{m} \dot{m} \dot{m} \dot{N}$
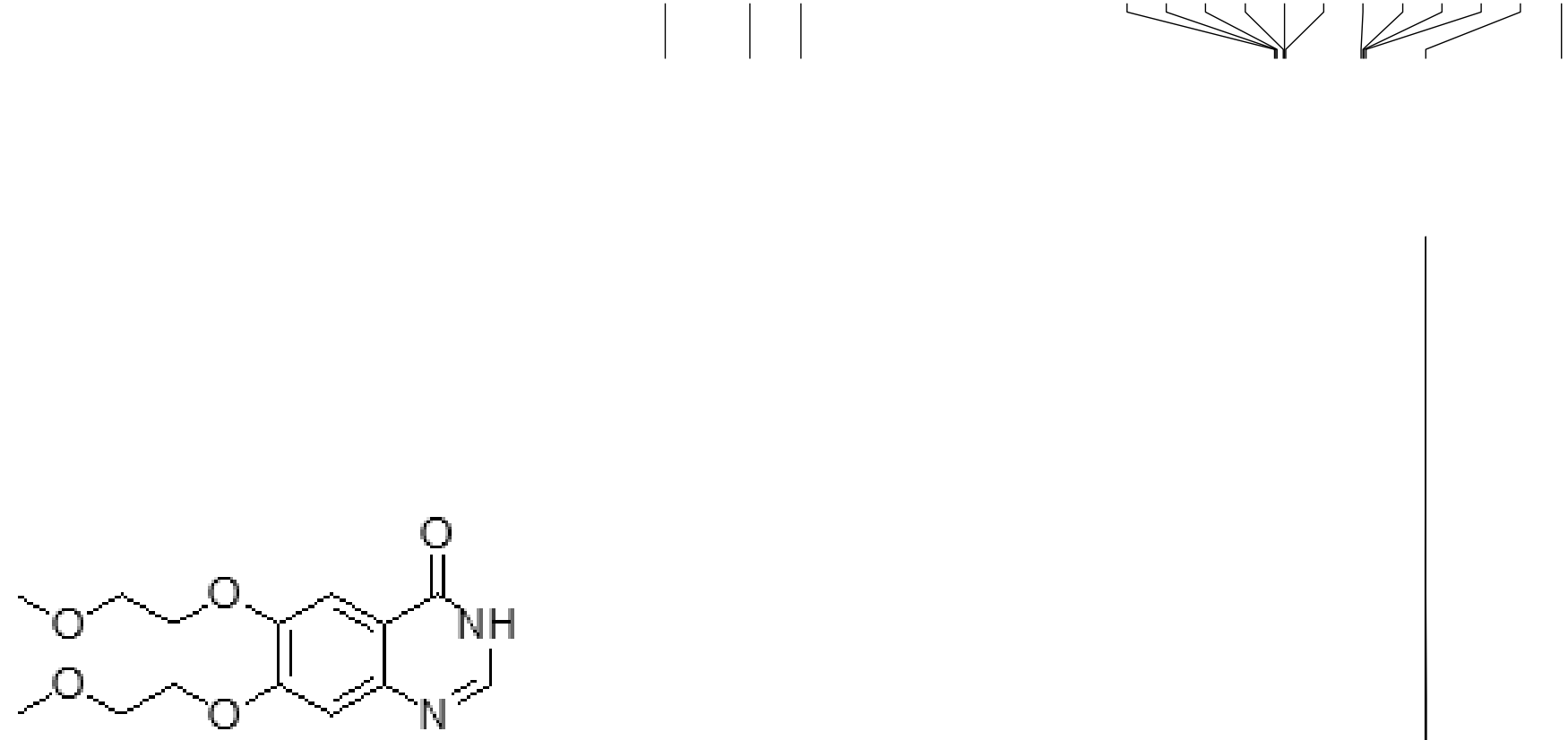

5
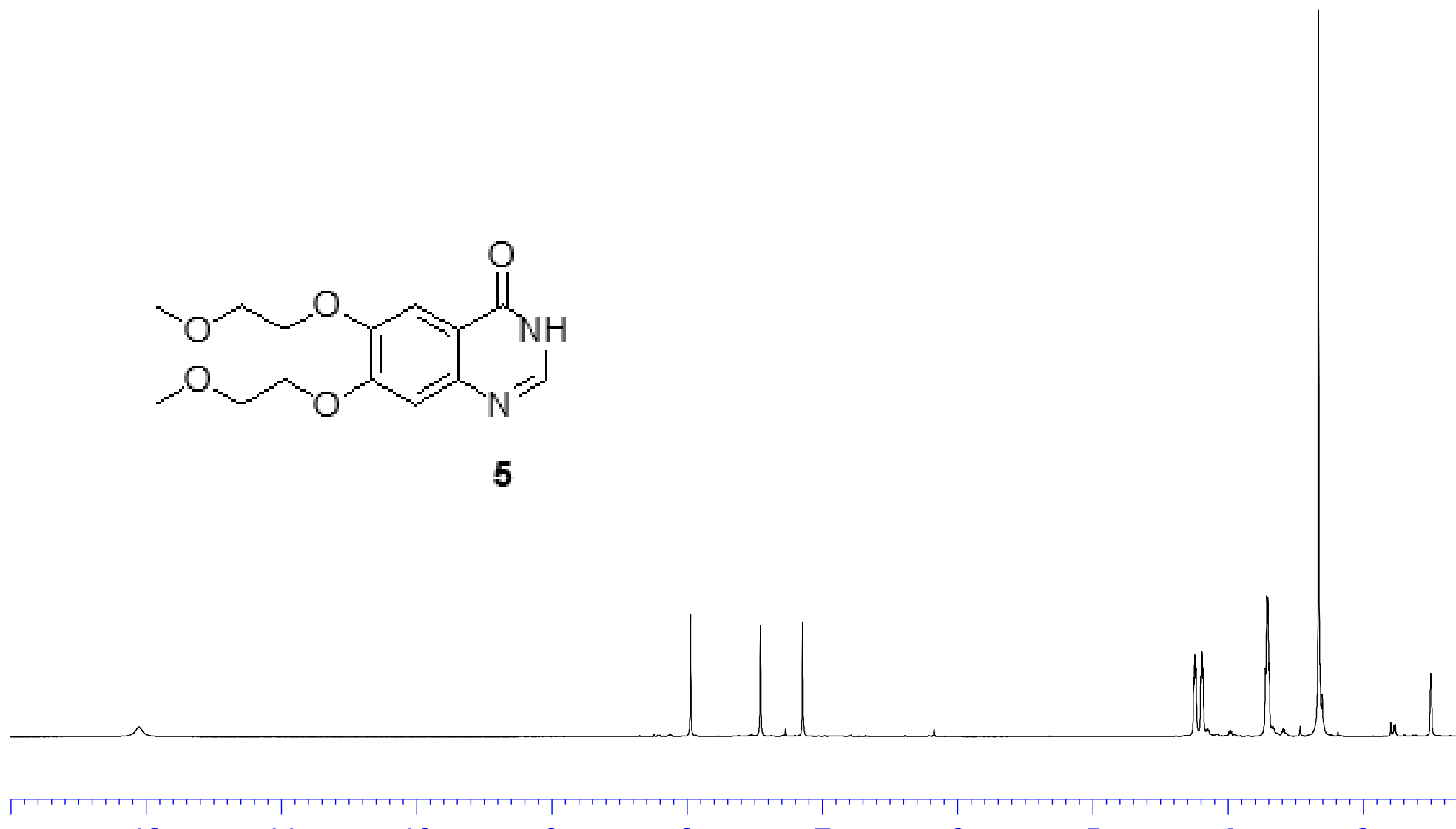

12

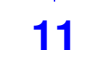

10

9

8

6

(bọ)

5

4

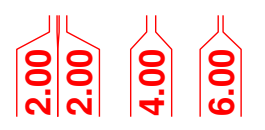



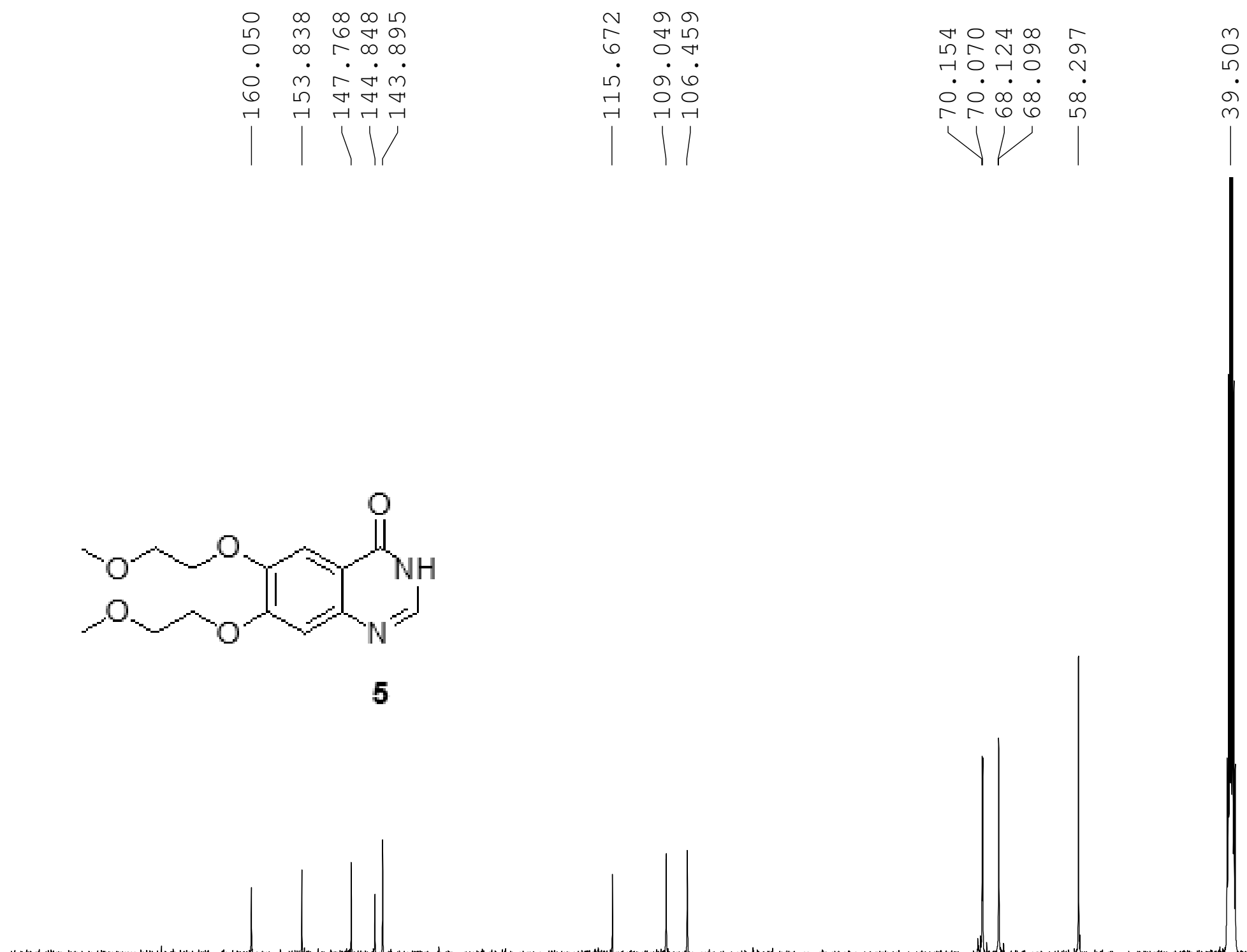
4-chloro-6,7-bis (2-methoxyethoxy) quinazoline Proton CDCl3

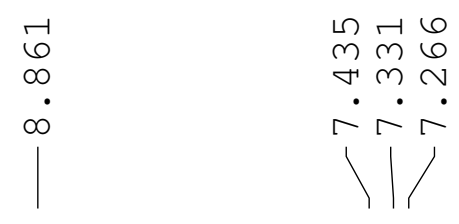

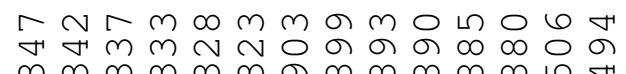

. $\cdot \cdot \cdot \cdot \cdot \cdot \cdot \cdot \cdot \cdot \dot{m} \cdot$

से अ
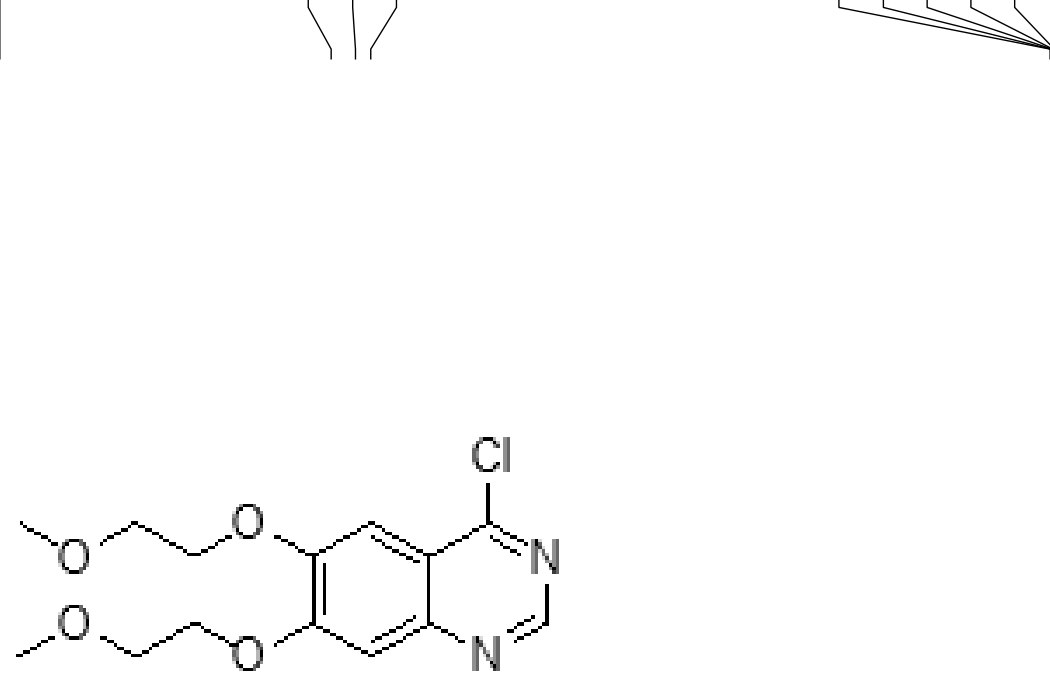

6
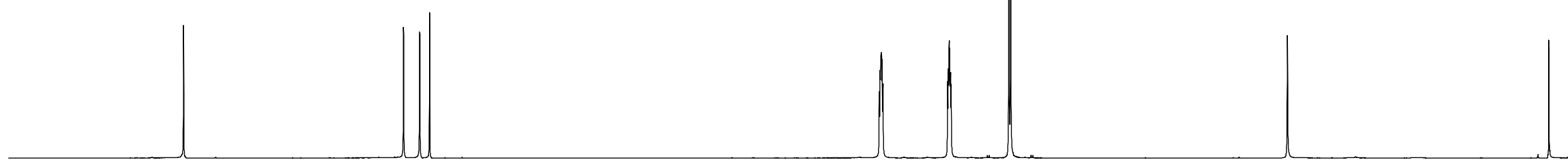

9

8

7

6

5

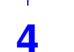

3

2

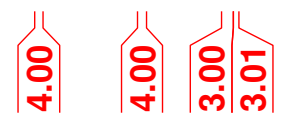

$\mid$

임영 

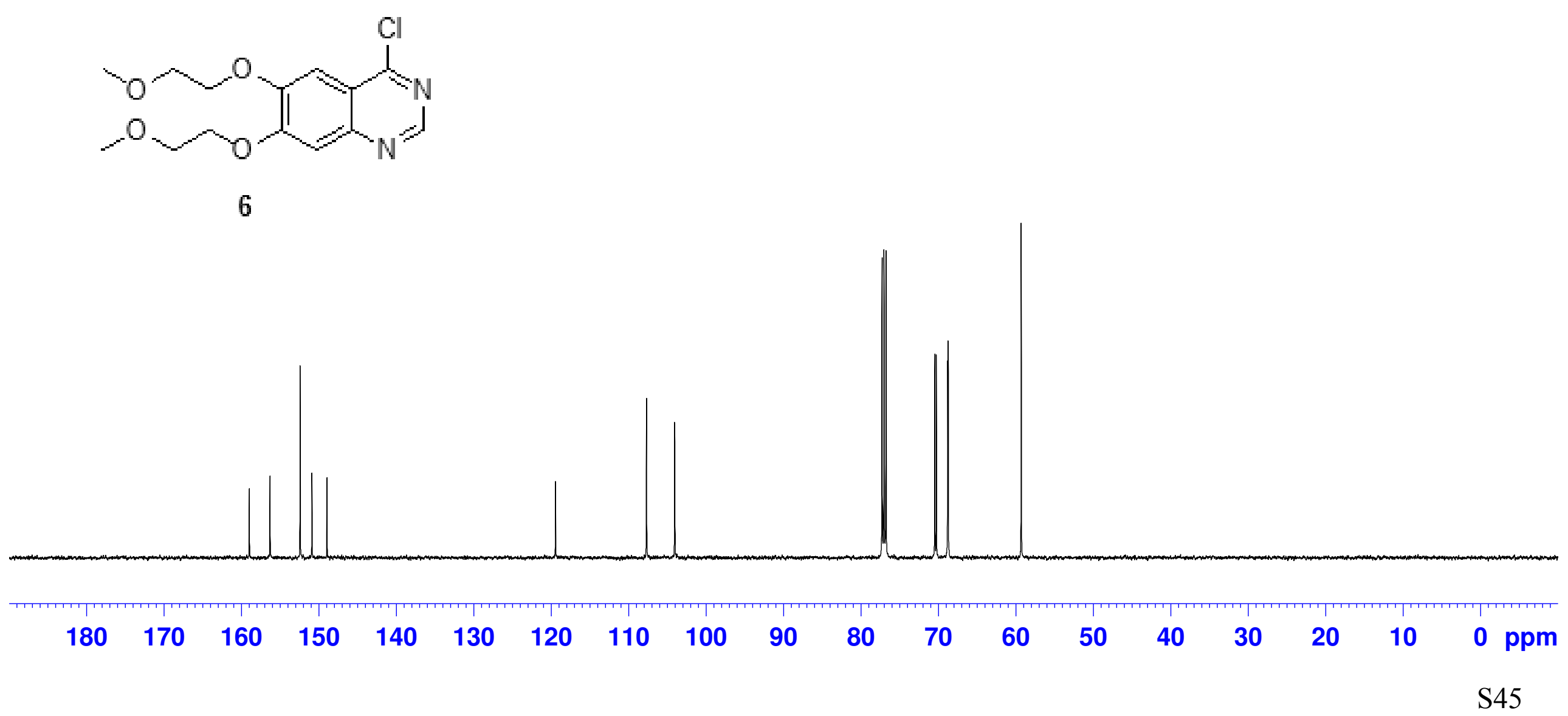
N-(3-ethynylphenyl)-6,7-bis (2-methoxyethoxy) quinazolin-4-amine Proton CDCl3

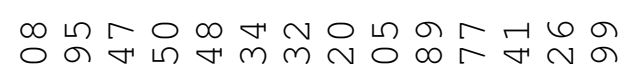
○ ब marararinarin. $\longrightarrow$

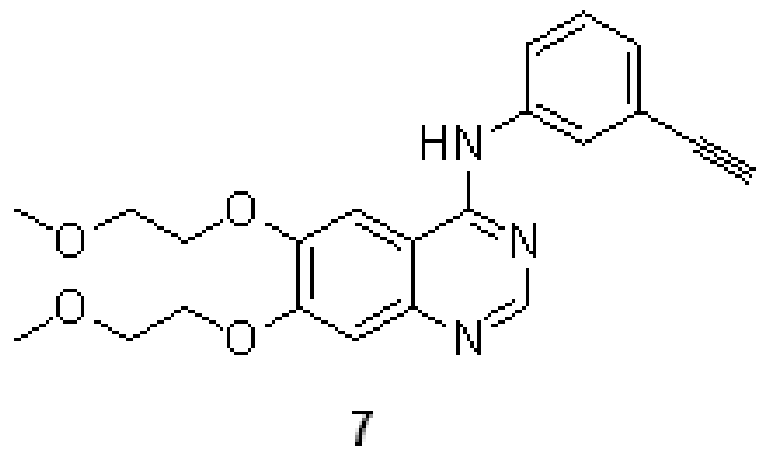

9

7

8
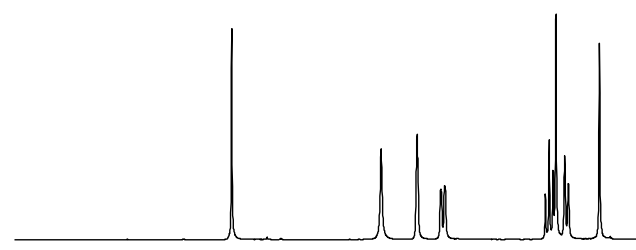

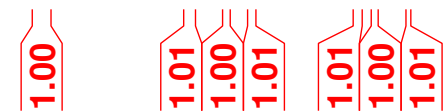

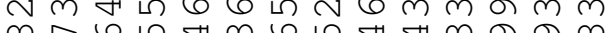

$\infty \pi, 4$

$\dot{r} \dot{r} \dot{r} \dot{r} \dot{r} \dot{m} \dot{m} \dot{m} \dot{m} \dot{m} \dot{m}$

$\longrightarrow$

5

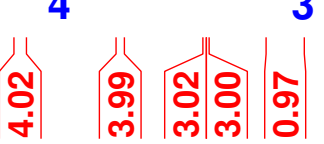

2 
N-(3-ethynylphenyl) -6,7-bis (2-methoxyethoxy) quinazolin-4-amine C13CPD CDCl3
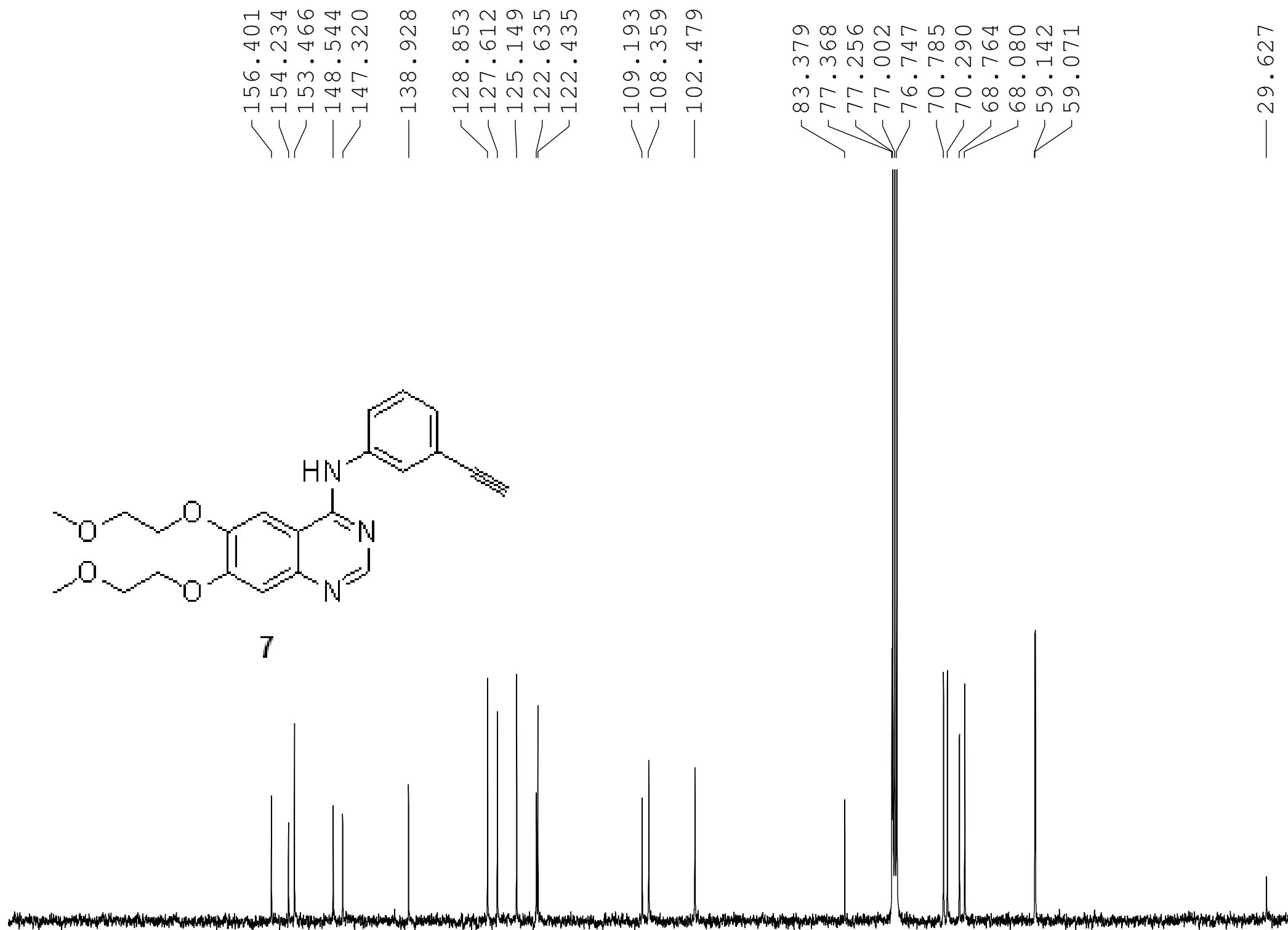

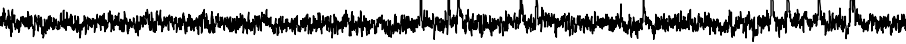




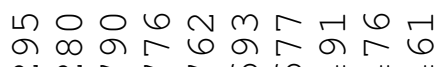

vor.

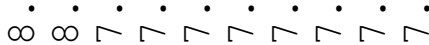

$\longrightarrow$
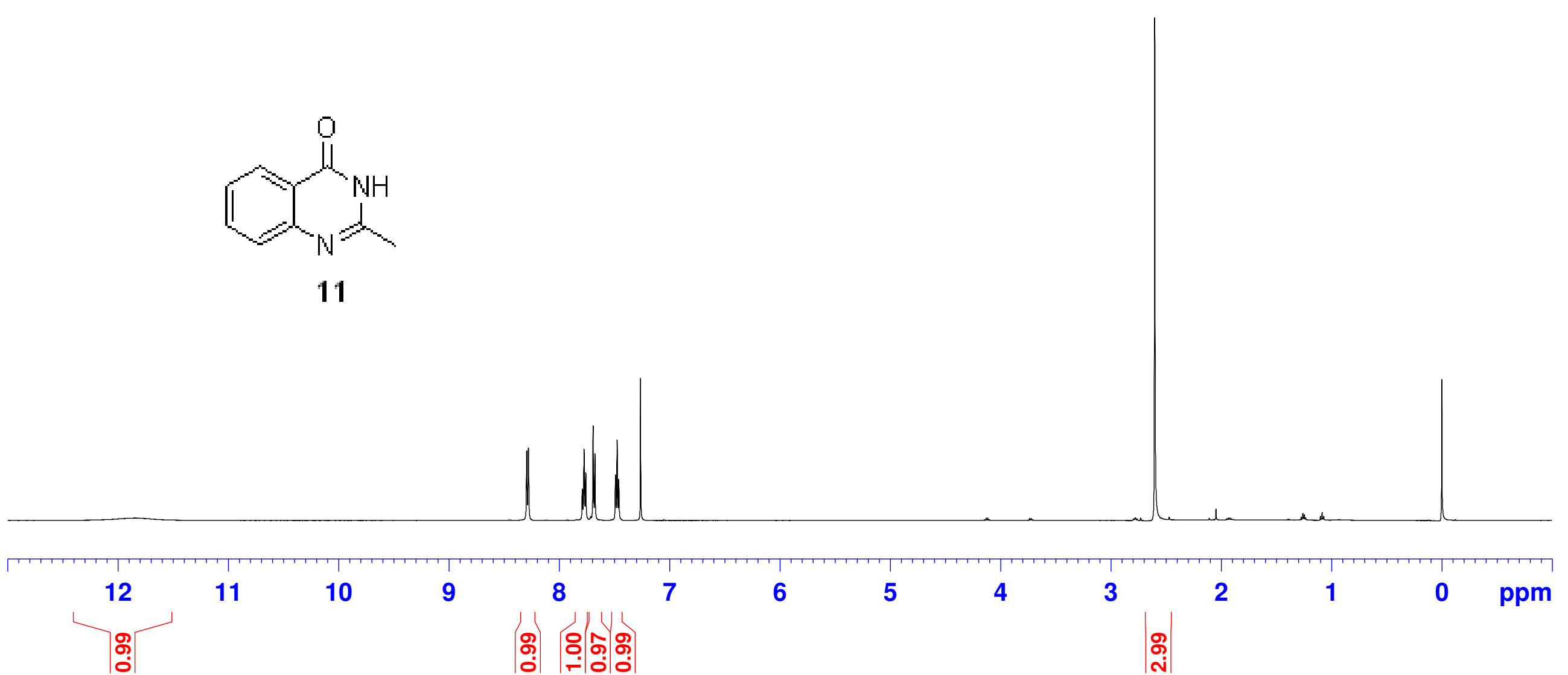
<smiles>Cc1nc2ccccc2c(=O)[nH]1</smiles>

11

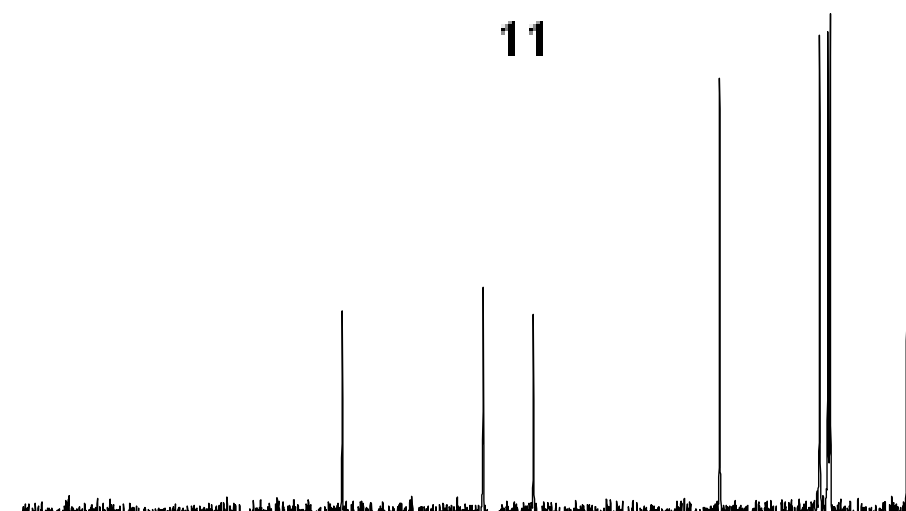

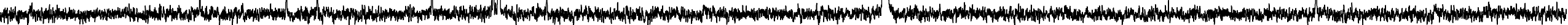



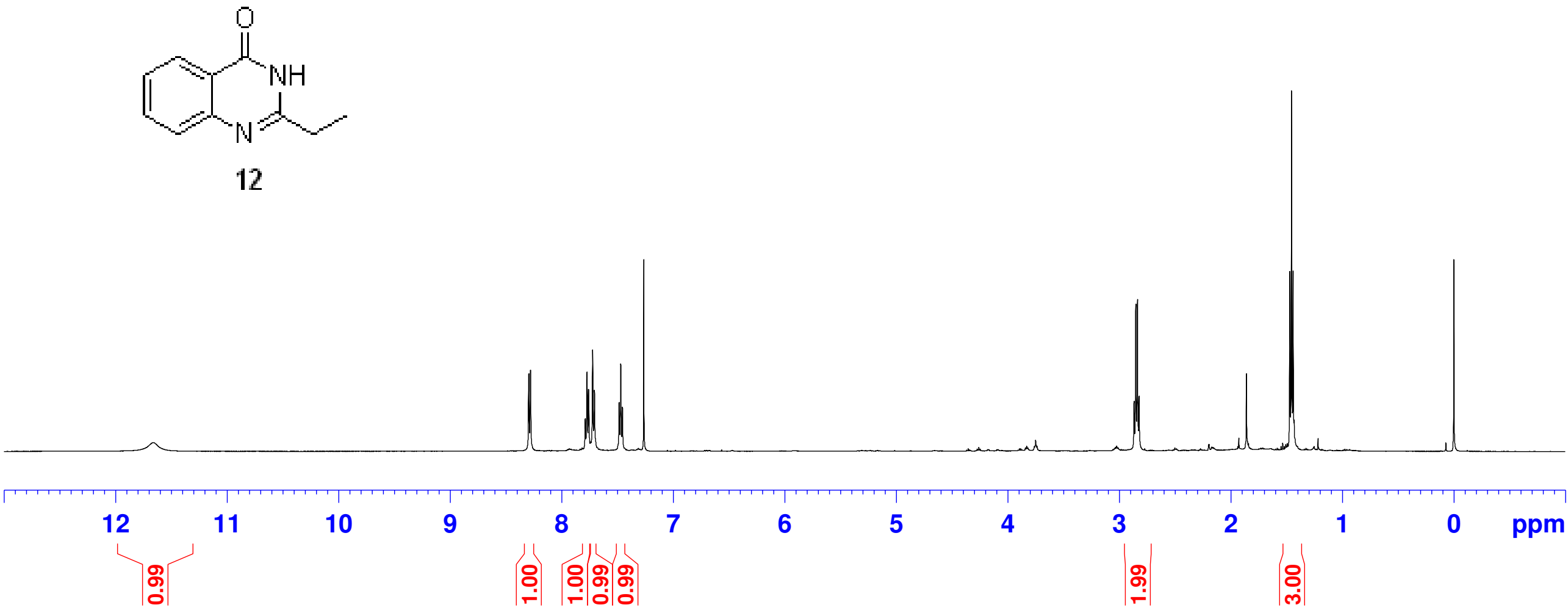

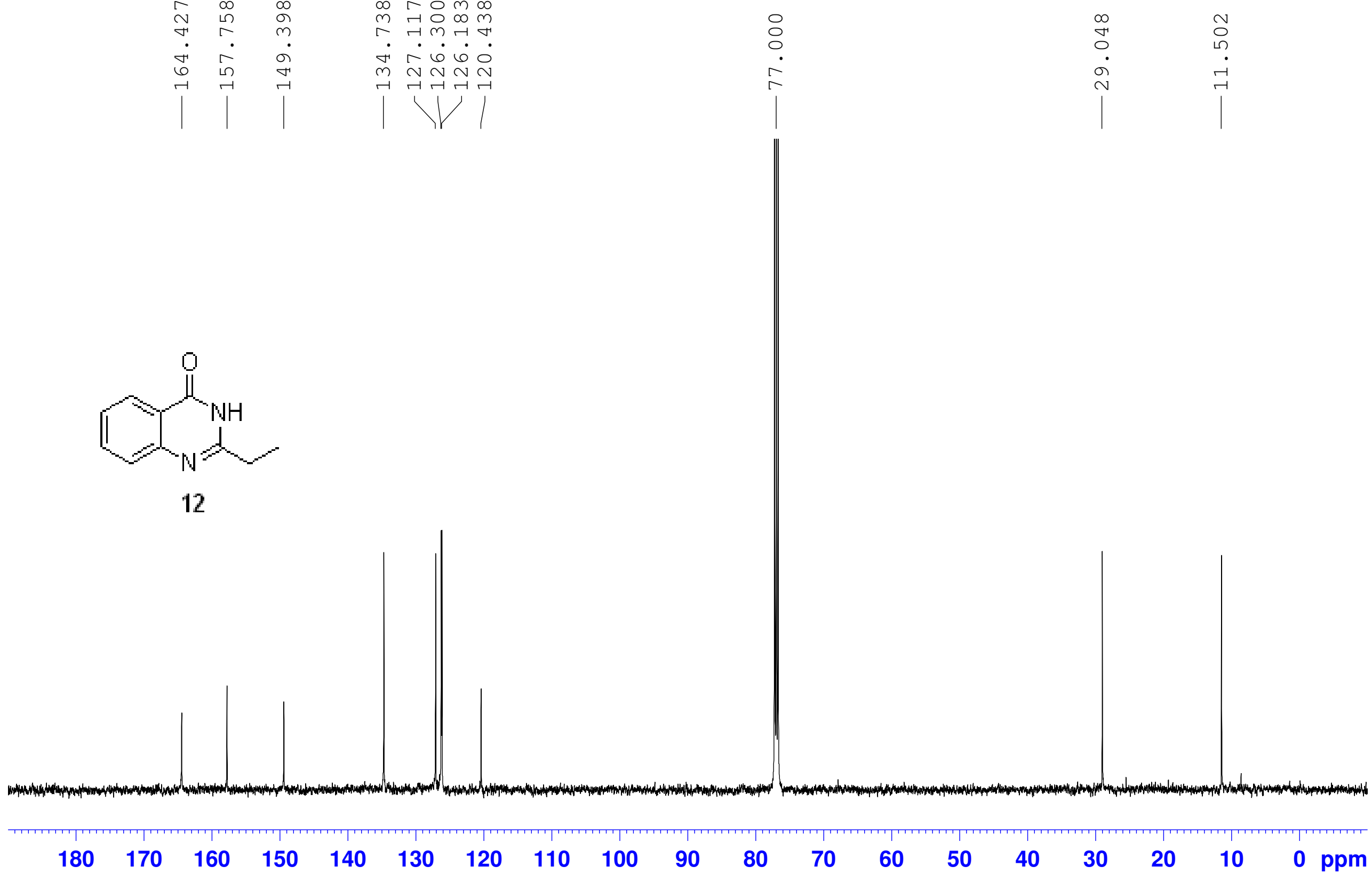

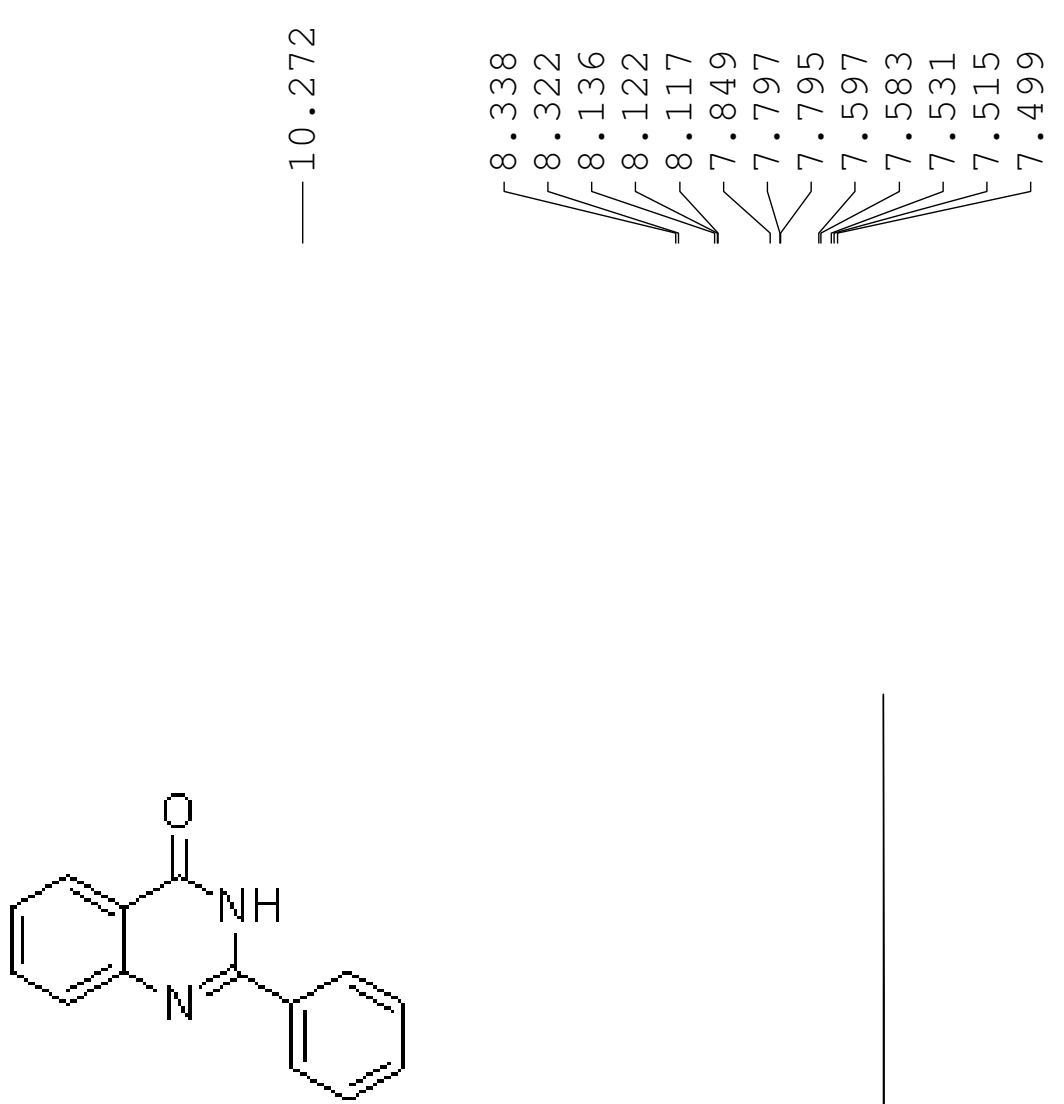

13

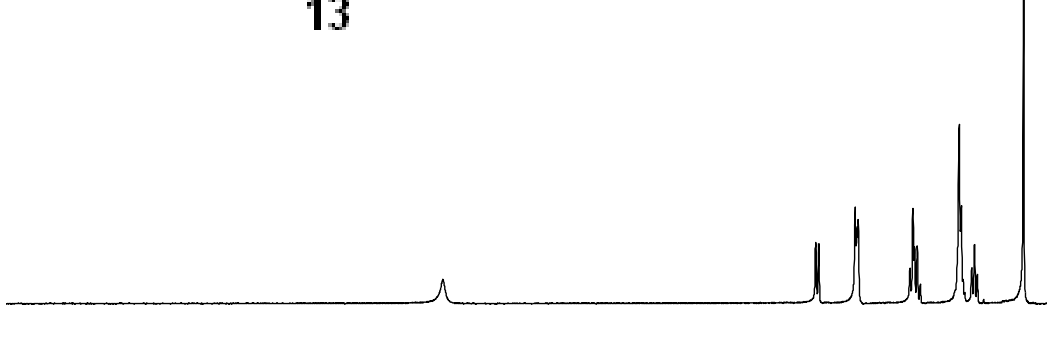


<smiles>O=c1[nH]c(-c2ccccc2)nc2ccccc12</smiles>

13

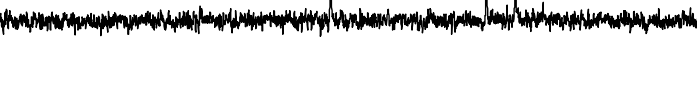

